Research Paper

\title{
Homoharringtonine inhibited breast cancer cells growth via miR-18a-3p/AKT/mTOR signaling pathway
}

\author{
Li-bin Wang ${ }^{*}$, Dan-ni Wang ${ }^{1,2^{*}}$, Li-gang $\mathrm{Wu}^{1}$, Jia Cao ${ }^{1}$, Jin-hai Tian ${ }^{1}$, Rong Liu ${ }^{1}$, Rong Ma ${ }^{1}$, Jing-jing $\mathrm{Yu}^{1}{ }^{1}$ Jia \\ Wang1, Qi Huang ${ }^{1}$, Wen-yong Xiong ${ }^{1,3 凶}$ and Xu Zhang ${ }^{1 凶}$ \\ 1. The General Hospital of Ningxia Medical University, Biochip Research Center, Yinchuan, 750001, China. \\ 2. Gansu Provincial Hospital, Clinical Laboratory Center, Lanzhou, 730000, China. \\ 3. Key Laboratory of Medicinal Chemistry for Natural Resource, Yunnan University, Kunming, 650091, China. \\ *These authors contributed equally to this work. \\ $\square$ Corresponding authors: Xu Zhang and Wenyong Xiong. Xu Zhang, MD, The Biochip Research Center of General Hospital of Ningxia Medical University, 804 \\ Shengli Street, Yinchuan, Ningxia, 750001, China.Tel: +86-951-6744181, E-mail:xuzhang1012@163.com. Wenyong Xiong, MD, Yunnan University Key Laboratory \\ of Medicinal Chemistry for Natural Resource. No 2, Cuihu North Road, Kunming, Yunnan, 650091, China. Tel: +8618987098832, E-mail: xwy@ynu.edu.cn.
}

(1) The author(s). This is an open access article distributed under the terms of the Creative Commons Attribution License (https://creativecommons.org/licenses/by/4.0/). See http://ivyspring.com/terms for full terms and conditions.

Received: 2020.02.13; Accepted: 2021.02.03; Published: 2021.03.02

\begin{abstract}
Homoharringtonine (HHT), a natural alkaloid derived from the cephalotaxus, exhibited its anti-cancer effects in hematological malignancies clinically. However, its pesticide effects and mechanisms in treating solid tumors remain unclear. In this study, we found that $\mathrm{HHT}$ was capable of inhibiting tumor growth after 5-days treatment of breast cancer cells, MCF-7, in vivo. Furthemore, HHT also significantly inhibited the cancer cell growth and induced cell apoptosis in vitro. miRNA sequencing proved miR-18a-3p was noticeably downregulated in the cells after HHT treatment. Moreover, downregulating miR-18a-3p increased HHT-induced cell apoptosis; our data supported that HHT suppressed miR-18a-3p expression and inhibited tumorigenesis might via AKT-mTOR signaling pathway. In conclusion: our study proved that HHT suppressed breast cancer cell growth and promoted apoptosis mediated by regulating of the miR-18a-3p-AKT-mTOR signaling pathway, HHT may be a promising antitumor agent in breast cancer treatment.
\end{abstract}

Key words: HHT; breast cancer; miR-18a-3p; AKT-mTOR signal pathway

\section{Introduction}

Breast cancer is one of the leading causes of cancer-related deaths in women. The epidemiological survey showed that the incidence of breast cancer in the world is increasing year by year [1]. Most patients with this type of cancer are diagnosed at an advanced stage of the disease, and the prognosis of these patients is still in poverty [2]. At present, adjuvant chemotherapy and radiotherapy after surgical resection are the most commonly treatment strategies for breast cancer. However, multidrug resistance and toxicity of chemotherapy medicine have often influenced the outcome of the treatment [3, 4]. Although a variety of chemotherapeutic medicine and target therapy are currently used in clinical, the median survival of patients with metastatic breast cancer was not improved significantly [5, 6]. Therefore, to investigating new medicine for breast cancer treatment became urgent.
Homoharringtonine (HHT), an alkaloid which extracted from cephalotaxus, has been approved by the USA FDA for treatment of patients with hematological malignancies. HHT exerts its anti-tumor effects by fixing to ribosomes, disabling elongation of the nascent peptide chain and further protein synthesis $[7,8]$. It has proved that HHT could induce leukemia cell apoptosis by inhibiting expression of the apoptosis-related proteins [9]. Several studies indicated that HHT also has anticancer effects on solid tumors. Wang et.al reported that HHT induced apoptosis and inhibited STAT3 via IL-6-JAK1-STAT3 signaling pathway in lung cancer cells [10]. HHT also performed its anti-cancer activity by altering the characteristics of immune cells in the Kras mutation non-small cell cancer cells [11]. A recent study reported that HHT suppressed triple negative breast cancer growth through reducing the 
expression of apoptosis proteins [12]. The results indicated that HHT could be a potential therapeutic medicine for breast cancer. However, the anti-cancer effects of HHT on breast cancer and the underlying mechanisms are not clear yet.In this study, we investigated the effects of HHT on human breast cancer cells and studied the underlying molecular mechanism. We provided evidences that HHT inhibited breast cancer cell growth and induced apoptosis by regulating the miR-18a-3p-AKT-mTOR signaling pathway, supporting the HHT could be applied in breast cancer treatment in future.

\section{Materials and Methods}

\section{Clinical samples collection}

The study obtained approval from the Ethics Committee of General Hospital of Ningxia Medical University, which was conducted in accordance with the Declaration of Helsinki. Six paired breast cancer tissues and adjacent non-cancerous tissue samples were collected from the department of oncology.

\section{Cell culture and transfection}

Homoharringtonine (HHT) was purchased from Hangzhou Minsheng Pharmaceutical Co, Ltd (Hangzhou, China). Human breast cancer cell lines MDA-MB-231, MCF-7, T47D, HCC1937 and MCF-10A were purchased from the American Type Culture Collection (ATCC, Mannasas,VA,USA). The cells were routinely maintained in Dulbecco's modified Eagle's medium (DMEM, HyClone, Logan, UT, USA), including 10\% fetal bovine serum (FBS, Invitrogen, Carlsbad, CA, USA) and 1\% penicillin-Streptomycin Solution at $37{ }^{\circ} \mathrm{C}$ in a humidified atmosphere containing $5 \% \mathrm{CO}_{2}$. miR-18a-3p lentivirus inhibitor (miR-18a-3p-inhibitor) and negative control virus (NC) was ordered from Genechem Company (Shanghai, China). $20 \mu \mathrm{M}$ miR-18a-3p-inhibitor or NC were transfected into the cells at $\sim 50 \%$ density using HitransG P Transfection Reagent (Genechem, Shanghai) according to the manufacturer's protocol guide.

\section{BrdU staining}

$1 \times 10^{4}$ cells were cultured in 24-well plates overnight at $37{ }^{\circ} \mathrm{C}, 5 \% \mathrm{CO}_{2}$. DMEM medium with different concentration of HHT as added to each well, and cells were cultured at $37^{\circ} \mathrm{C}$ for 2 days. Add 10 $\mu \mathrm{g} / \mathrm{ml}$ BrdU (Abcam, USA, and incubation for $2 \mathrm{~h}$. The cells were then washed with PBS and fixed in $4 \%$ paraformaldehyde for $30 \mathrm{~min}$. Subsequently, $2 \mathrm{M} \mathrm{HCl}$ were used to treat the cells $30 \mathrm{~min}$ at room temperature. After being washed three times with PBS buffer, the cells were treated with $0.5 \%$ Triton X-100 and then blocked with 5\% goat serum
(ZSGB-Bio, Beijing, China) for $1 \mathrm{~h}$. The cells were incubated with a monoclonal rat primary antibody against BrdU (1:1000) at $4{ }^{\circ} \mathrm{C}$ overnight,after being washed three times with PBS buffer and followed by incubation with Alexa FluorR® 488 goat anti-rat IgG secondary antibody (H+L; 1:250, Invitrogen). The nuclei were stained with DAPI (1:500). The percentage of BrdU staining was calculated from at least 10 microscopic fields.

\section{Cell proliferation and colony formation assays}

The cell proliferation ability was assessed with CCK-8 and colony formation assay. For the CCK-8 assay, after treated with difference concentration of HHT or infected miR-18a-3p inhibitor or NC, the cells were cultured for $24 \mathrm{~h}, 48 \mathrm{~h}$ and $72 \mathrm{~h}$ separately. Then treated the cells with CCK-8 reagent (KeyGEN BioTECH, Jiangsu, China) and further cultured for $2 \mathrm{~h}$ according to the manufacturer's instructions. The optical density was measured using the spectrophotometer (Glomax Multi Detection Systerm, Promega, USA) at $450 \mathrm{~nm}$. Each group of experiments included five replicates and repeated three times.

For the colony formation assay, $8 \times 102$ cells were cultured in 6-well plate. The cells were exchanged medium every three days. After 15 days, discarded medium, fixed the plates with $100 \%$ methanol for 15 min and then stained with crystal violet for $10 \mathrm{~min}$. The number of colonies was observed and calculated. All experiments were performed in triplicate.

\section{Wound-healing and Transwell assay}

The cells were seeded in 12-well plate with DMEM medium for $48 \mathrm{~h}$. Then the cell monolayer was wounded by $200 \mu$ l pipette tip scraping. The exfoliated cells were washed with PBS and continue cultured for additional $24 \mathrm{~h}, 48 \mathrm{~h}, 72 \mathrm{~h}$ with different concentrations of HHT, transfected miR-18a-3p inhibitor or NC respectively. The speed of wound closure was observed and photographed using a microscope (Nikon, Tokyo, Japan). Cell mobility was assessed by measuring three randomly perpendicular wound width.

For the transwell assay, Corning Incorporated Transwell Chambers (Corning, $8 \mu \mathrm{m}, \mathrm{NY}$, USA) were used to detect the migration capacity of the cells. Placed the chambers into the 24-well plate that contained culture medium supplemented with $20 \%$ FBS as a chemo-attractant, $2 \times 10^{4}$ cells were suspended in serum-free medium and loaded into the upper chamber. Cells were incubated at $37^{\circ} \mathrm{C}$ and allowed to invade through the membrane pores. After $15 \mathrm{~h}$, the non-invading cells were removed and the lower side of the membrane were fixed with 100\% methanol and stained with crystal violet. The cells were 
photographed under a microscope and the cell migration was observed to calculate the number of transmembrane cells.

\section{Cell apoptosis detection}

The cells were seeded in 6-well plates and treated with different concentration of HHT, transfected miR-18a-3p inhibitor or NC for $48 \mathrm{~h}$. Cells were harvested and washed with cold PBS and then resuspended in $500 \mu \mathrm{L}$ of binding buffer (BestBio, Shanghai, China). Incubate the cells with $5 \mu \mathrm{L}$ Annexin V-FITC (BestBio, Shanghai, China) and $10 \mu \mathrm{L}$ Propidium lodide (BestBio, Shanghai, China) at room temperature for $15 \mathrm{~min}$ in the dark. Samples were examined using BD flow cytometer and the data were analyzed with FlowJo software (TreeStar Corporation, Ashland, OR, USA).

\section{Western blotting}

After treating with different concentration of HHT, tranfected miR-18a-3p inhibitor or NC, the cells were harvested in the RIPA lysis buffer (Beyotime Biotechnology, Shanghai, China). The total protein was extracted and the concentration of each sample was determined by BCA protein assay reagent kit (Thermo Fisher Scientific, Inc.). The supernatants containing total protein were mixed with a corresponding volume of $5 \times$ SDS loading buffer (Beyotime Biotechnology, Shanghai, China) and heated at $100{ }^{\circ} \mathrm{C}$ for $10 \mathrm{~min}$. The lysates of $30 \mu \mathrm{g}$ were electrophoresed on $10 \%$ SDS-PAGE and transferred to PVDF membranes. The proteins were blocked containing 5\% defatted milk for $2 \mathrm{~h}$ at room temperature. Subsequently, primary antibodies were incubated at $4^{\circ} \mathrm{C}$ overnight. Followed by incubation with conjugated secondary antibodies (anti-rabbit, 1:10,000, cat: \#7074, or anti-mouse, 1:10,000, cat: \#7076, CST, Inc.) for $1.5 \mathrm{~h}$ at room temperature. To ensure equal amounts of sample protein applied for electrophoresis, GAPDH was used as an internal control.

\section{RNA isolation and qRT-PCR}

Total RNA was extracted from patient tissue and plasma samples using the Trizol reagent (Invitrogen) and purified with miRNA Isolation Kit (Ambion, Austin, TX, USA) according to the protocol. The purity and concentration of RNA were determined from OD260/280 readings using spectrophotometer NanoDrop2000 (Thermo Fisher Scientific). RNA integrity was determined by $1 \%$ formaldehyde denaturing gel electrophoresis.

The cDNA was synthesized by the Superscript Reverse Transcription System (Invitrogen). RT-qPCR was performed using TB Green qPCR Mastermix (TaKaRa, Japan) on LightCycler ${ }^{\circledR} 480$ real-time PCR
Platform (Roche). The qRT-PCR reaction in a total volume of $20 \mu \mathrm{L}$ system, including $0.8 \mu \mathrm{L} / 10 \mu \mathrm{M}$ forward/reverse primers, $10 \mu \mathrm{L}$ TB Green qPCR Mastermix, $2 \mu \mathrm{L}$ cDNA, and $6.4 \mu \mathrm{L}$ double-distilled water. The cycling program is $95{ }^{\circ} \mathrm{C}$ for $30 \mathrm{sec}$, followed by 40 cycles of $95{ }^{\circ} \mathrm{C}$ for $8 \mathrm{sec}$ and a pre-selected annealing temperature for $30 \mathrm{sec}$. The primers (Table 1) were synthesized Sangon biotech (Shanghai) Co. Ltd. The relative expression of genes were calculated using the $\Delta \Delta \mathrm{CT}$ method.

After treatment with $\mathrm{HHT}$ at $37^{\circ} \mathrm{C}$ for 2 days, the cells were harvested and total RNA was isolated using Trizol reagent (Invitrogen, CA, USA) according to the manufacturers' instructions. The purity and concentration of RNA were determined by using spectrophotometer NanoDrop2000 (Thermo Fisher Scientific), cDNA synthesis was performed according to the reverse transcription kit (TaKaRa, Shanghai, China) manufacturer's protocols. The PCR amplification was performed with specific primers and carried out using the SYBR-Green PCR system (Takara Bio, Inc). GAPDH served as internal control. Calculation of the relative expression of each gene was quantified by the $2^{-\Delta \Delta \mathrm{Ct}}$ method.

Table 1. The prime sequence for RT-qPCR

\begin{tabular}{llll}
\hline Gene Name & Primer Sequence $\left(5^{\prime} \rightarrow 3^{\prime}\right)$ & $\begin{array}{l}\text { miRBase Accession } \\
\text { number }\end{array}$ \\
\hline hsa-miR-18a-3p & F & CGACTGCCCTAAGTGCTCC & MIMAT0002891 \\
& R & AGTGCAGGGTCCGAGGTATT & \\
hsa-miR-363-3p & F & GCGAATTGCACGGTATCCA & MIMAT0000707 \\
& R & AGTGCAGGGTCCGAGGTATT & \\
hsa-miR-21-3p & F & GCGCAACACCAGTCGATG & MIMAT0004494 \\
& R & AGTGCAGGGTCCGAGGTATT & \\
hsa-miR-375-3p & F & CGGGTTTGTTCGTTCGGCT & MIMAT0000728 \\
& R & GTGCAGGGTCCGAGGTATT & \\
\hline
\end{tabular}

The prime sequence designed for RT-qPCR, the primer were synthesized by Sangon biotech (Shanghai) Co. Ltd, China.

Annotation: F for Up prime; R for Down prime.

\section{microRNA sequencing and analysis}

RNA samples were submitted to CapitalBio Technology (Beijing, China) for microRNA sequencing and analysis. The data read from highthroughput sequencing was first screened, then 18 to $30 \mathrm{nt}$ clean reads are mapped to the Rfam database (http://rfam.janelia.org/) and the Repbase database (http://girinst.org/repbase/), short RNA maps to miRNA precursors of the human reference genome, and mature miRNAs are deposited into the miRBase 21.0 database (http://www.mirbase.org/). Using the existing mirdeep2 software to identify the minimum free energy of the Dicer lysate and the unannotated small RNA marker in the previous step by exploring the secondary structure, and predicting new candidate miRNAs for unannotated short reads not mapped to miRBase 21.0 miRNA expression levels 
were estimated by TPM (transcript per million). Differential expression analysis of two flowering phases was performed using the $\mathrm{R}$ package edge $\mathrm{R} /$ limma. miRNAs that had changed ratios of more than 2 or less than 0.5 (Fold change $\log _{2}>1$ or $<-1$ ) and $P<0.05$ was set as the threshold for significant differential expression by default. Target gene prediction of differential expression miRNAs was performed using the miRanda. A GO functional enrichment analysis and KEGG pathway enrichment analysis were performed for Target gene using the KOBAS 3.0 software (http://kobas.cbi.pku.edu.cn). GO terms and pathway terms with $P$-value less than 0.05 were considered significantly enriched by target genes.

Differentially expressed genes were validated by quantitative real-time PCR (qPCR). The primer sequences for the miR-18a-3p and U6 were listed in supplements Table 1.The data were normalized using the U6 transcriptsfor miRNA. The relative expression of each gene was quantified by the $2-\Delta \Delta \mathrm{Ct}$ method.

\section{In vivo tumorigenicity assay}

5-6 week old female BALB/c male nude mice (bought from Animal Center of the Chinese Academy of Science, Shanghai, China) were housed and maintained under specific pathogen-free conditions following animal ethic guideline of the Animal Care and Use Committee of Ningxia Medical University. $2 \times 10^{7}$ MCF-7 cells were injected subcutaneously into each mouse. Treatment was initiated when tumors reached a volume of $50-150 \mathrm{~mm}^{3}$. Tumor volume was calculated as the largest length $\times$ width was $2 \times 0.5$ and tumor diameter was measured three times every week. Then divided the mice into control group and HHT treatment group, each group contained seven animals. HHT was diluted in PBS and was intraperitoneally injected with a daily dose of $50 \mathrm{\mu g} / \mathrm{kg}$ for 10 days. The animals were sacrificed and tumor mass was collected for further study.

\section{Statistical analysis}

Statistical analyses were performed using SPSS23.0 software (IBM, USA) and GraphPad Prism 7.0 (GraphPad Software, USA). All assays were conducted three times and data were presented as mean \pm SD. Statistical differences were determined by $t$-test. The results were considered significant when $P$ values $<0.05$.

\section{Results}

\section{HHT inhibited breast cancer cells growth}

To investigate the effect of HHT on breast cancer cell growth, 4 breast cancer cell lines (MDA-MB-231, HCC-1937 MCF-7 and T47D) were used to examine the effects. After treated with a series of concentrations of HHT for $48 \mathrm{~h}$, the inhibition rates of proliferation of all tested cells were significantly increased in a dose-dependent manners (Fig. 1a), demonstrating that HHT has a broad anti-cancer effects on human breast cancer cells. In addition, the IC50 of HHT for each breast cancer cell line showed that MDA-MB-231 and MCF-7 cells were more sensitive to HHT compared to other cell lines (Table 2). Therefore, we selected these two cells for subsequent experiments. CCK-8 assays revealed that HHT significantly inhibited MDA-MB-231 and MCF-7 cell growth in a dose-dependent manner and time-dependent manner (Fig. 1b, c). BrdU staining assay showed that HHT significantly decreased the percentage of BrdU positive cells on MDA-MB-231 and MCF-7 cells in a dose-dependent manners (Fig. $1 d$ ). Colony formation assays were performed to further investigate the effect of HHT on the self-renewal capability of the cells. The results showed that the colony numbers were smaller and fewer when treated with HHT (Fig. 1e). These results demonstrated that HHT remarkably inhibited breast cancer cells growth.

Table 2. The IC50 of HHT on different breast cancer cells

\begin{tabular}{ll}
\hline The cells & IC50 $(\mu \mathrm{g} / \mathrm{ml})$ \\
\hline MDA-MD-231 & 0.313 \\
HCC1937 & 0.324 \\
MCF-7 & 0.459 \\
T47D & 1.270 \\
\hline
\end{tabular}

The inhibitory concentration rate (IC50) of HHT on different breast cancer cells was detected by CCK-8 assay. The results showed MDA-MB-231 and MCF-7 cells were more sensitive to HHT compared to other cell lines.

\section{HHT inhibited migration and invasion of breast cancer cells}

The effects of HHT on the migration and invasion abilities of breast cancer cells were also evaluated via transwell and wound-healing assay. The results showed that the cell scratch healing rate was significantly lower than that of the control group when treated with HHT. This experiment was consistent with the transwell experiment results, indicating that HHT can inhibit the migration ability of breast cancer cells. The statistical results are showed as representative histograms (Fig. 2a).

Compared with the control cells, the cell invasion ability was significantly inhibited when treated with HHT. The number of invasion cells of MDA-MB-231 and MCF-7 was obviously decreased. The invasion inhibition of HHT on the cells was in a dose-dependent manner (Fig. 2b). We further evaluated the expression of FAK and MMP9 by Western blot assays. The results showed that the 
expression of two proteins was deregulated after treatment with HHT (Fig. 2c). The results suggested
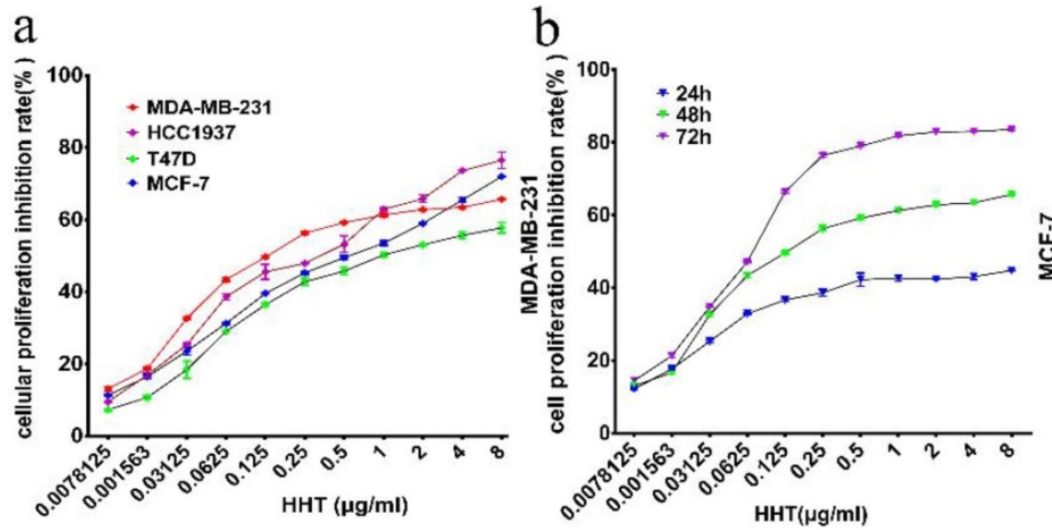

HHT
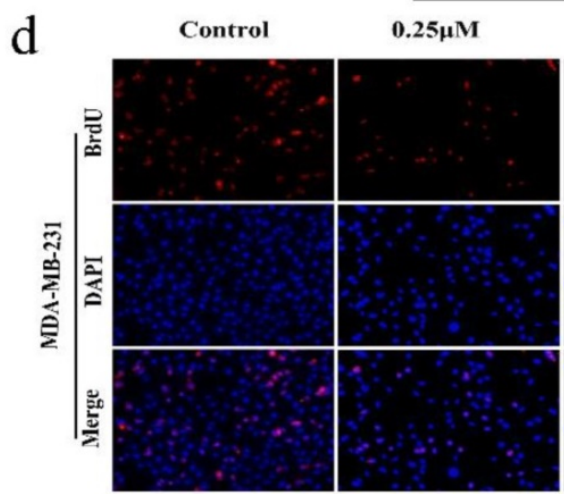

$0.5 \mu \mathrm{M}$
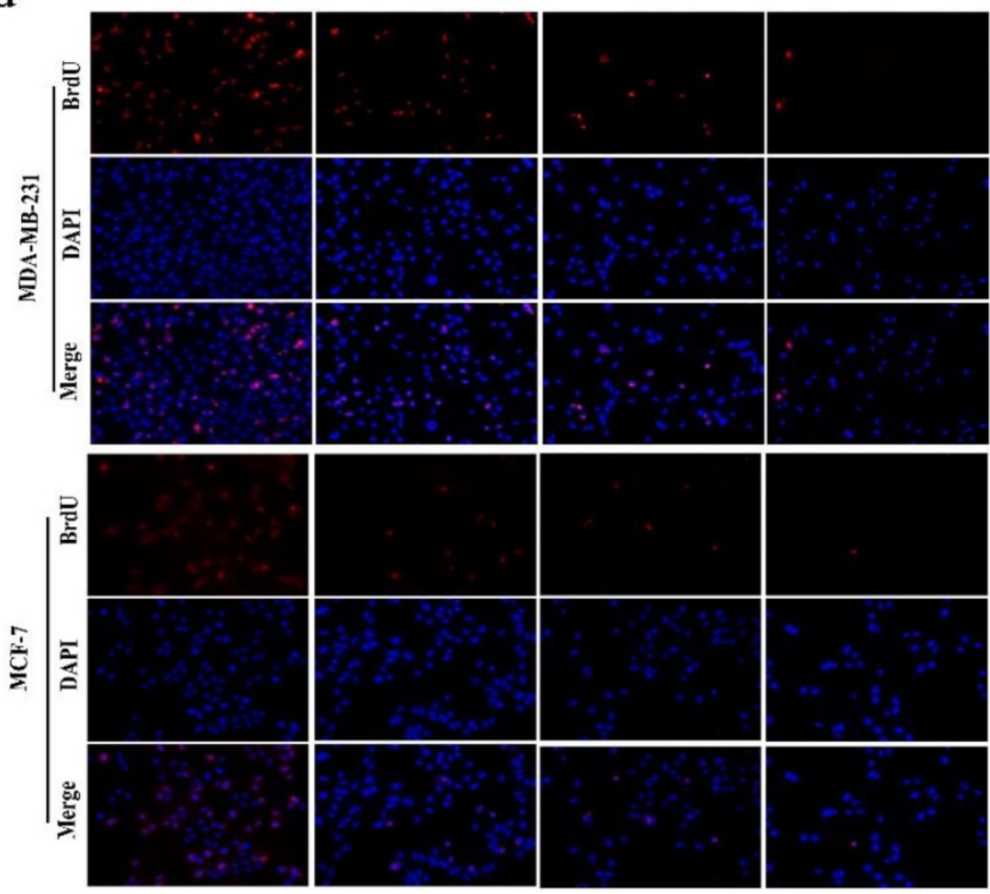

e
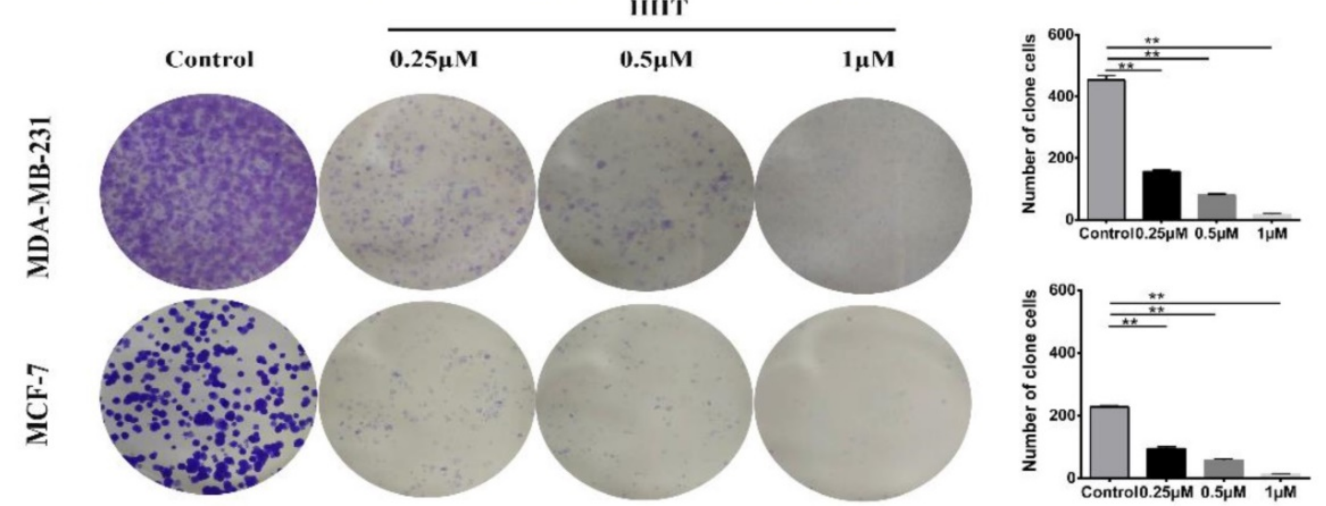

Figure 1. HHT inhibited breast cancer cells growth and proliferation. a. Effects of different concentrations of HHT on breast cancer cells, including MDA-MB-231, MCF-7,T47D and HCC1937 cells, while screening drug-sensitive cell lines. b. MDA-MB-231 cell was treated with different concentrations of HHT for $24 \mathrm{~h}, 48 \mathrm{~h}$ and $72 \mathrm{~h}$. c. MCF-7 cell was treated with different concentrations of HHT for $24 \mathrm{~h}, 48 \mathrm{~h}$ and $72 \mathrm{~h}$. Cellular proliferation inhibition rate was evaluated using CCK-8 assays, and the data are presented as the means \pm SD. d. Images of MDA-MB-231 and MCF-7 cells positive for BrdU staining after treatment with indicated concentration of HHT, Scale bar $=100 \mu \mathrm{m}$. The histogram demonstrates the results of BrdU-positive rate in MDA-MB-231 and MCF-7 cells. e. Colony formation assays were used to investigate the colony formation abilities of MDA-MB-231 and MCF-7 cells after treatment with the indicated concentrations of HHT. All data were analysed using unpaired Student's t-tests and are shown as the means \pm SD. $* p<0.05, * * p<0.01$. 
a

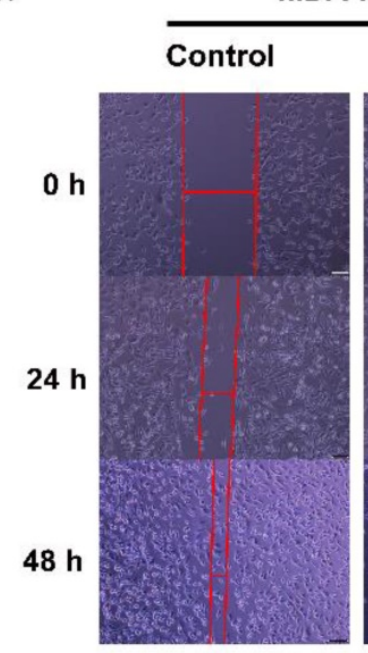

MDA-MB-231

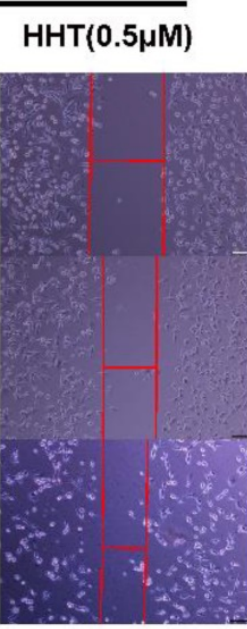

MCF-7

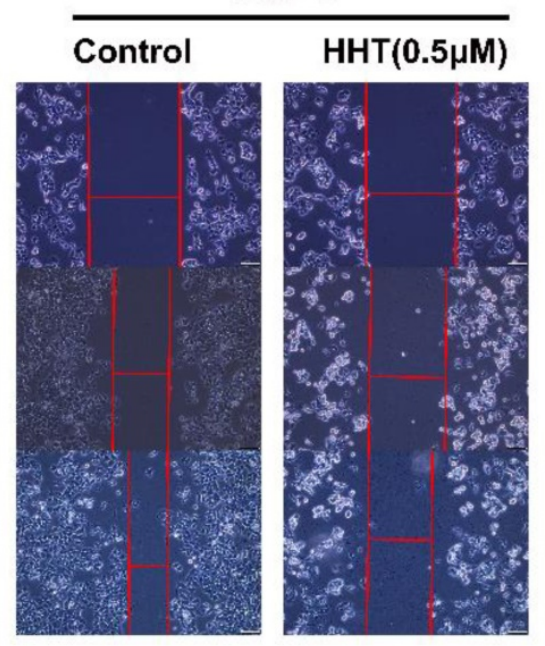

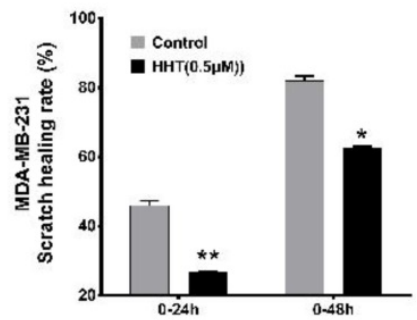

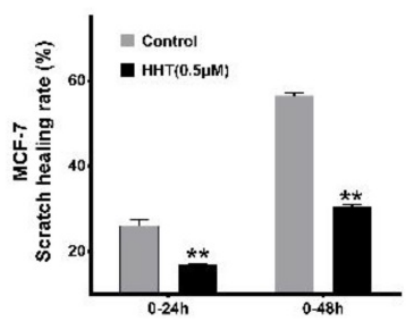

$\mathrm{b}$

\section{Migration}
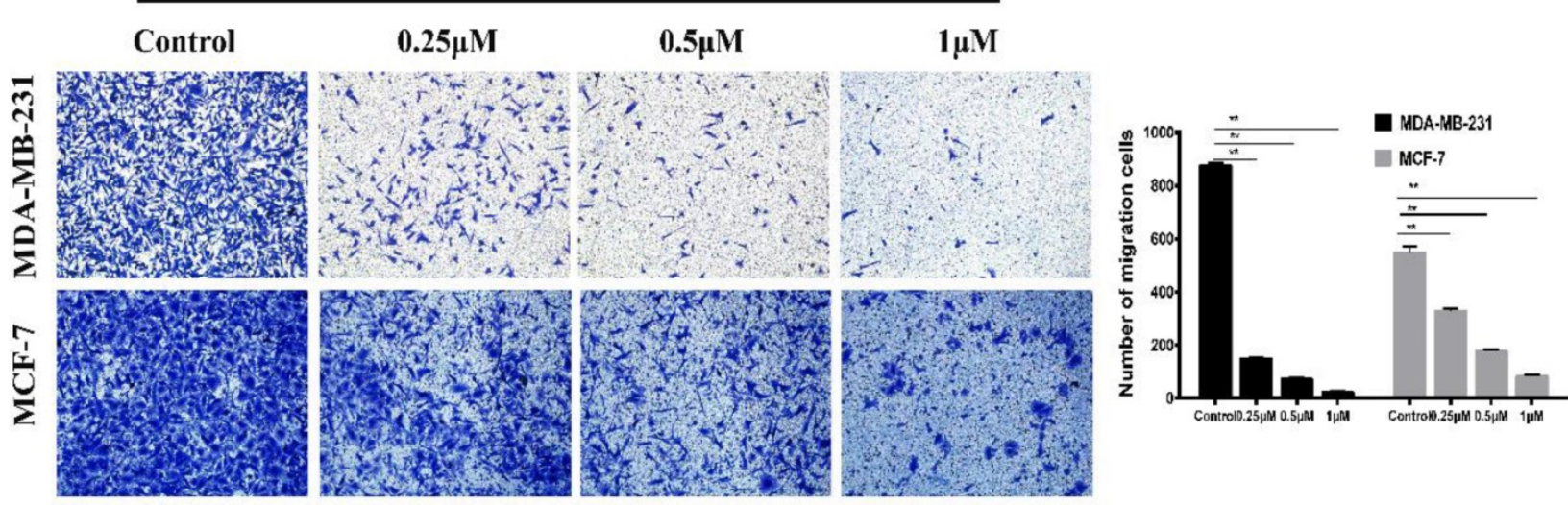

C
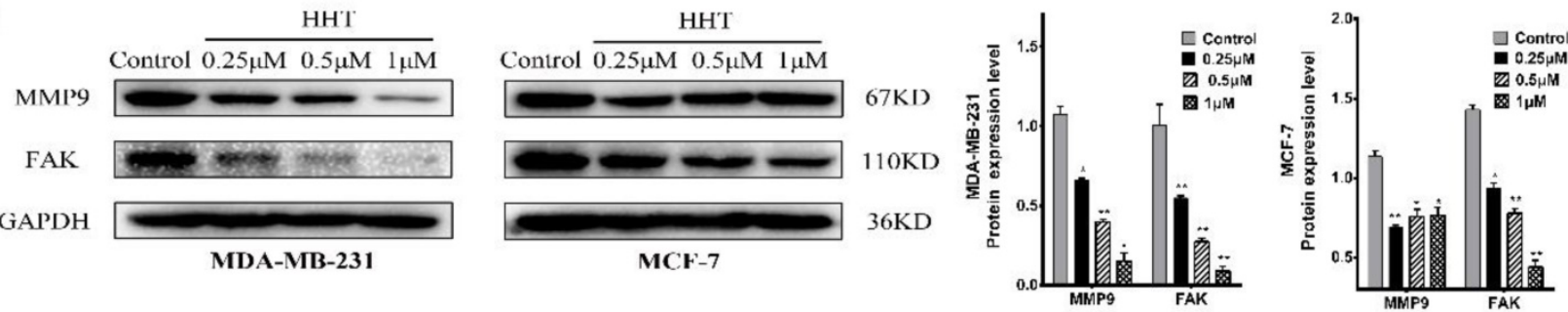

Figure 2. HHT inhibited migration and invasion abilities of breast cancer cells. a. In vitro migration ability of MDA-MB-231 and MCF-7 cells were detected using scratch wound healing assay after different concentrations of HHT for $48 \mathrm{~h}$. b. Transwell migration assays of MDA-MB-231 and MCF-7 cells treated with HHT. c. The expression of MMP9 and FAK were analysed by Western blot. GAPDH was used as the control. All data were analysed using unpaired $t$-tests and are shown as the means \pm SD. $* 2<0.05$, ${ }^{*} \mathrm{p}<0.01$.

\section{HHT induced breast cancer cells apoptosis}

With AnnexinV/ PI staining and flow cytometry analysis, we evaluated the effects of HHT on the cells apoptosis. The results demonstrated that the apoptosis of MCF-7 and MDA-MB-231 cells being induced by HHT in a dose-dependent manner (Fig. 3a). The Western bloting showed that the expression of Bax, cleaved-caspase 3, cleaved-caspase 9, and cleaved PARP protein were increased when treated with $\mathrm{HHT}$, while $\mathrm{Bcl} 2$, pro-caspase 3, pro-caspase 9, and PARP protein expression were decreased (Fig. $3 b)$.

\section{HHT suppressed miR-18a-3p expression in breast cancer cells}

To investigate the mechanism of HHT induced breast cancer cell apoptosis. High-throughput sequencing was used for microRNA sequencing in the cells with HHT treatment. A total of 379 miRNA candidate was selected based on the screening criteria as a fold-change $>1.5$ or $\leq 1.5$ and a $P$-value $<0.05$. We then narrowed the analysis scope and verified 30 aberrantly expressed miRNAs, including 15 upregulated and 15 downregulated (Fig. 4a). Quantitative real-time PCR validated that miR-18a- 
$3 p$, miR-21-3p and miR-363-3p were significantly lowexpressed in the HHT treatment group, while miR375-3p expression increased (Fig. 4b). Considering miR-18a-3p was high expression in 6 paired breast cancer tissues and the cell lines before HHT treatment (Fig. 4c, d). We proposed that miR-18a-3p might be the cause of HHT-inhibited breast cancer growth.

\section{Downregulating miR-18a-3p enhanced HHT functional in breast cancer cells}

Based on previous hypothesis, the miR-18a-3p inhibit lentivirus vector (miR-18a-3p inhibitor) being constructed and transfected into the breast cancer cell lines (Fig. 5a). miR-18a-3p expression was successfully decreased in MDA-MB-231 and MCF-7 cells treated by miR-18a-3p inhibitor. Furthemore, miR-18a-3p inhibitor, the expression of miR-18a-3p in the cells were significantly decreased (Fig. 5b). CCK-8 and clone formation assays verified the same conclusion (Fig. 5c, d, e). Wound healing and transwell assays were also performed to detect the effect of miR-18a-3p on breast cancer cells. The results revealed that inhibition of miR-18a-3p enhances the effect of HHT on breast cancer cells invasion and metastasis (Fig. 6a, b). Western blot proved that the metastasis associated proteins MMP9 and FAK expression were inhibited in the miR-18a-3p inhibitor group, and inhibited expression of miR-18a-3p can enhance the function of HHT on breast cancer cells (Fig. 6c). The results were consistent with cell

$\mathbf{a}$
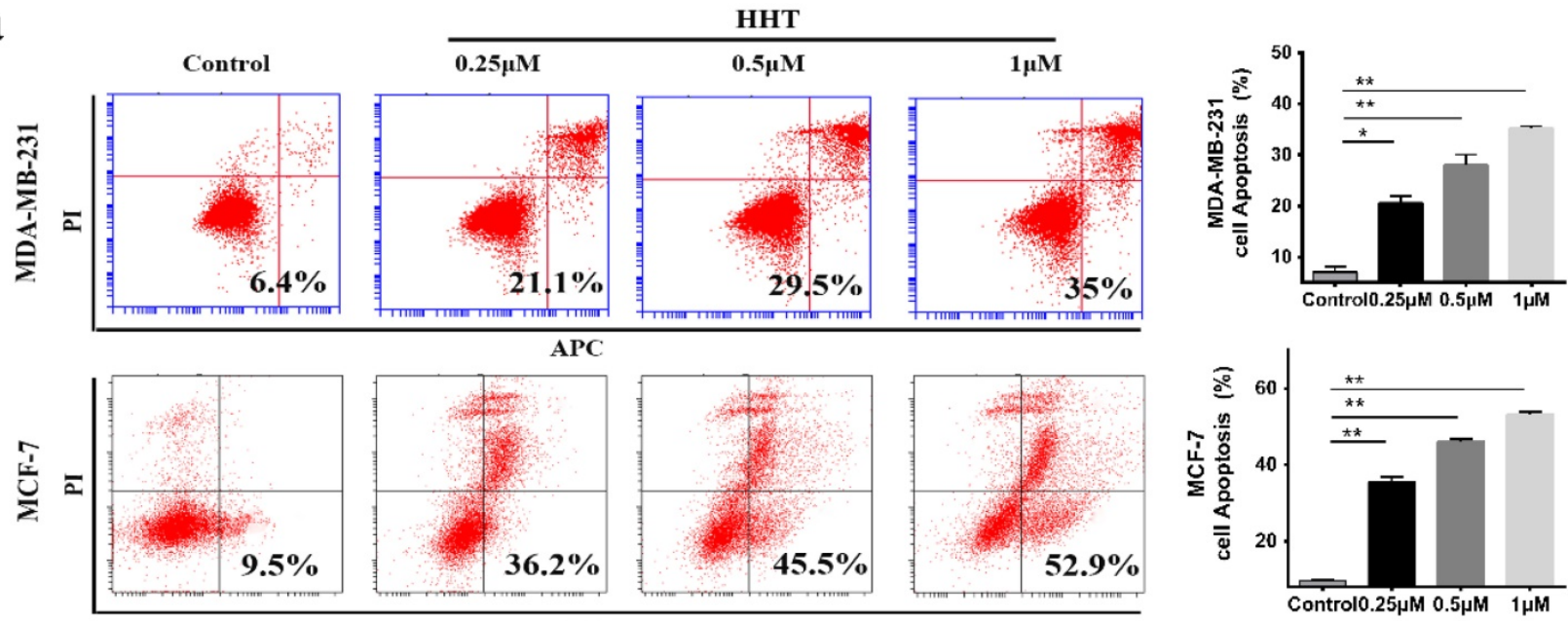

b
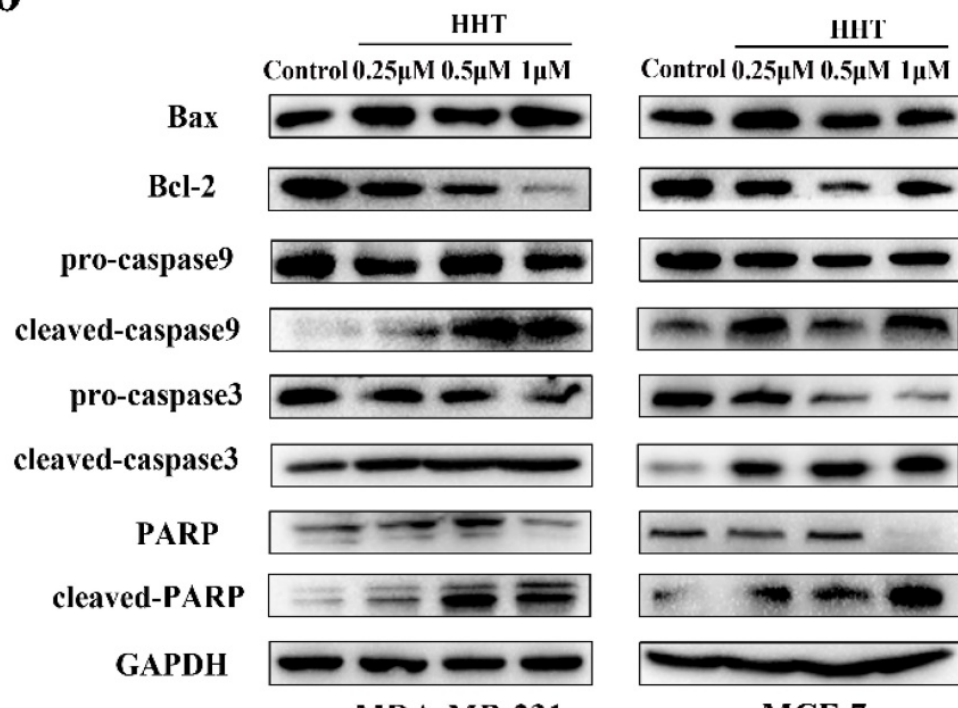

$20 \mathrm{KD}$

MDA-MB-231

MCF-7

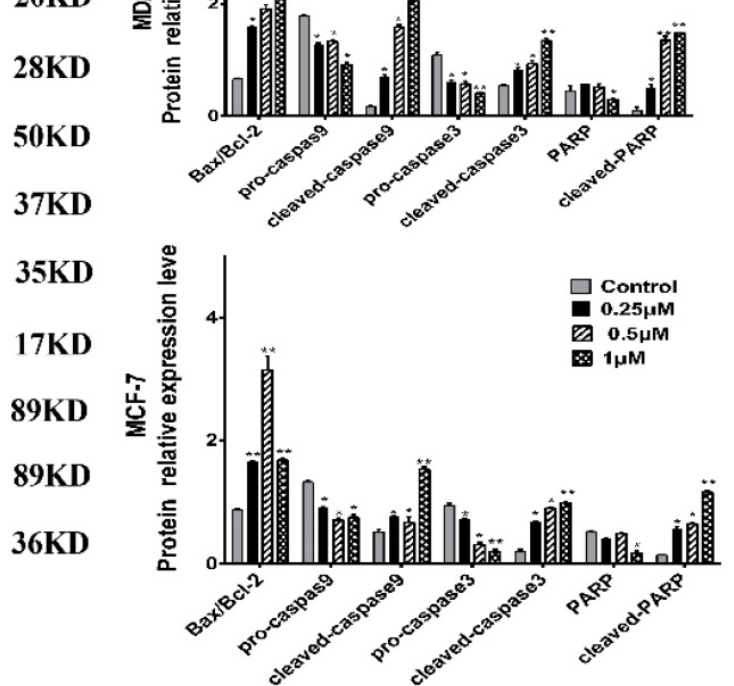

Figure 3. HHT induce breast cancer cells apoptosis. a. MDA-MB-231 and MCF-7 cells were treated with HHT for $48 \mathrm{~h}$, which caused a significant increase the proportion of apoptosis cells in a dose-dependent manner via flow cytometry. b. Western blot analysis of apoptosis regulatory-proteins after different concentrations of HHT for $48 \mathrm{~h}$. GAPDH was used as the control. All data were analysed using unpaired t-tests and are shown as the means \pm SD. ${ }^{*} p<0.05$, $* * p<0.01$. 
phenotypic experiments. Taken together, these data proved that miR-18a-3p plays an important role in regulating the biological characteristics of breast cancer cells with HHT treatment.

Flow cytometry indicated that the proportion of apoptotic cells in the miR-18a-3p inhibitor group was markedly increased. Downregulation of miR-18a-3p expression enhances HHT-induced apoptosis in breast cancer cells Simultaneously (Fig. 7a). Western blot showed that the expression of $\mathrm{Bcl} 2$, pro-caspase3, pro-caspase 9 and PARP were all decreased, while Bax, cleaved-caspase 3, cleaved-caspase 9, and cleaved PARP expression were increased. The results indicated that downregulation of miR-18a-3p expression enhanced HHT-induced apoptosis in breast cancer cells (Fig. 7b).

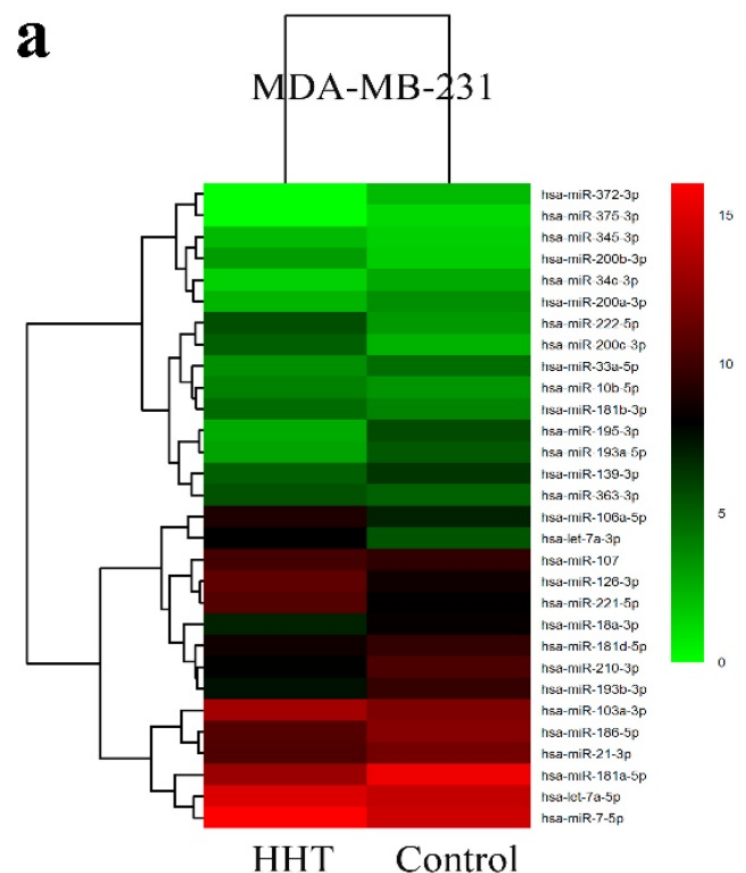

C

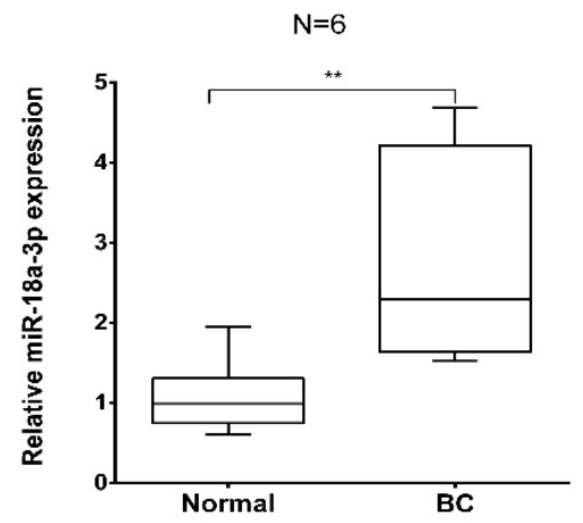

b
HHT inhibited breast cancer cells growth via miR-18a-3p-AKT-mTOR signaling pathway

The AKT-mTOR signaling pathway has been reported to regulate numerous cellular processes, such as cell growth, proliferation and migration [13, 14]. When analysis the relationship between the AKT-mTOR signaling pathway and miR-18a-3p, we found that AKT-mTOR signaling pathway were associated with miR-18a-3p expression (Fig. 8a). Western blot was conducted to identify the relationship and its influence for HHT treatment. The results showed that the expression of Rictor, PDK1, p-mTOR (ser-2481) and p-AKT (ser-473) were all decreased when treated the cell with HHT or miR18a-3p inhibitor. When combined treated the cells with HHT and miR-18a-3p inhibitor, the inhibition of
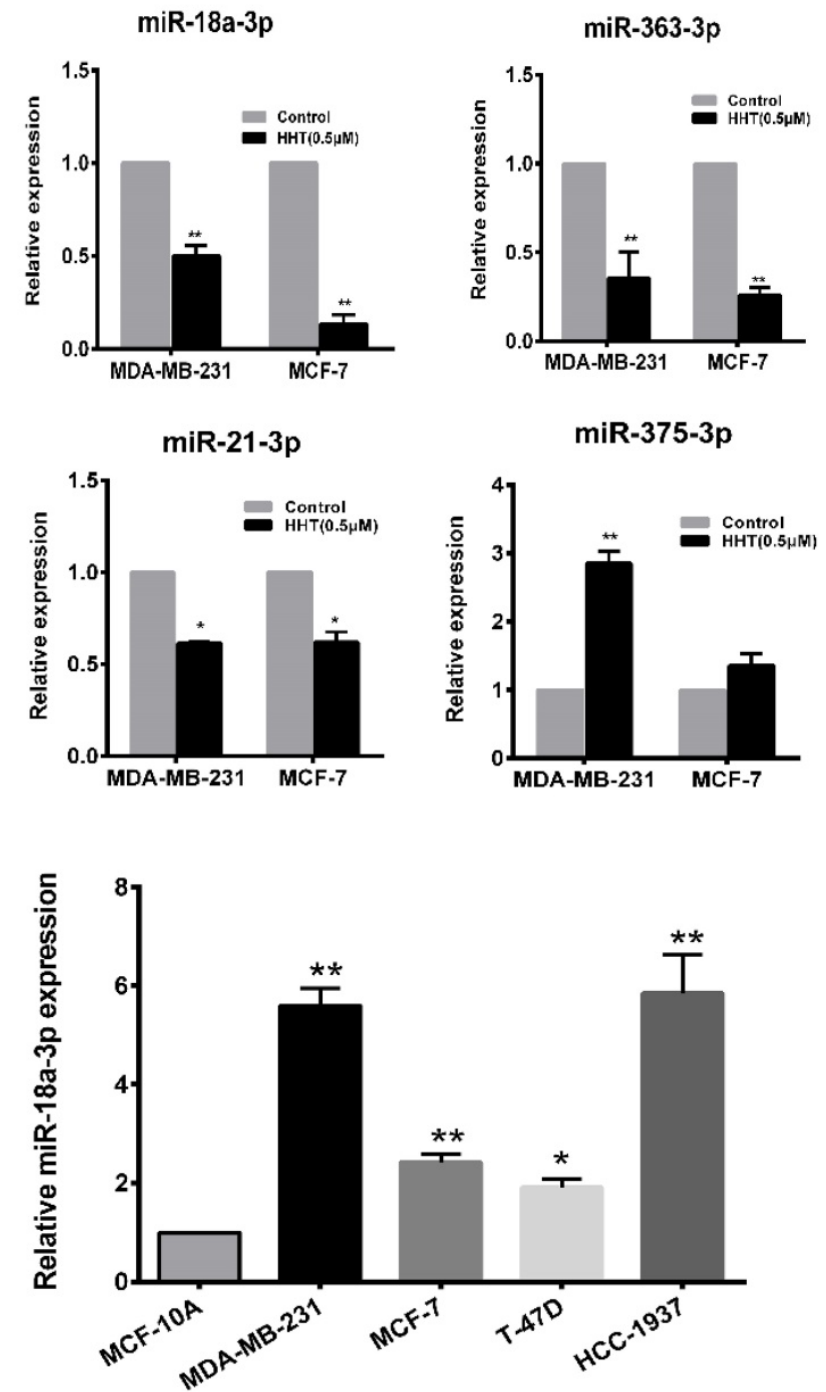

Figure 4. HHT suppressed miR-18a-3p expression in breast cancer cells. a. The heatmap shows the 30 significantly increased and decreased miRNAs in MDA-MB-23 after HHT treatment by small RNA sequencing. b. Relative expression of the 4 indicated miRNAs listed in (a) measured by RT-qPCR in MDA-MB-231 and MCF-7 cells. c. Relative expression of miR-18a-3p from 6 breast cancer tissues and adjacent non-tumor tissues. The expression levels of miR-18a-3p in 6 breast cancer cell lines and one normal breast epithelial cell line, MCF-10A. 
AKT-mTOR signaling pathway can be enhanced (Fig. $8 b)$. The results suggested there have a miR-18a-3pAKT-mTOR signaling pathway axis in BC cells. HHT suppressed breast cancer cell proliferation, migration and induced apoptosis through miR-18a-3p-AKTmTOR signaling pathway.

\section{HHT inhibits tumor growth in vivo}

To further validate the effects of HHT in vivo,
MCF-7 cells were injected subcutaneously into the BALB/c nude mouse. 10 days after injection, the mouse were divided into two groups and treated with PBS and $50 \mu \mathrm{g} / \mathrm{kg}$ HHT separately. After HHT treatment, the xenografts volume and weight were significantly lower than those in the PBS group (Fig. $9 \mathrm{a}-\mathrm{c})$. The findings suggested that HHT has functional of inhibited tumor growth in vivo. Immunochemistry staining results proved that caspase-3, Bax expression a

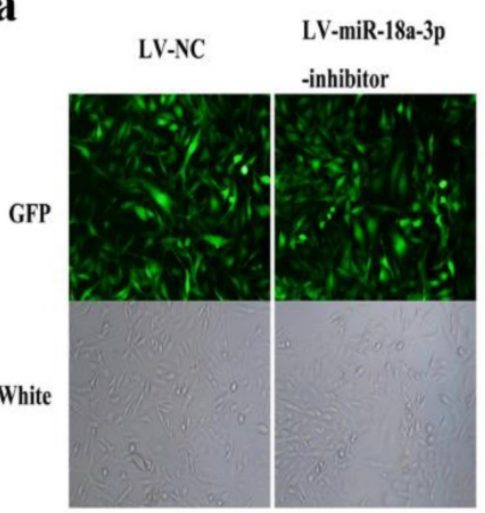

MDA-MB-231

c

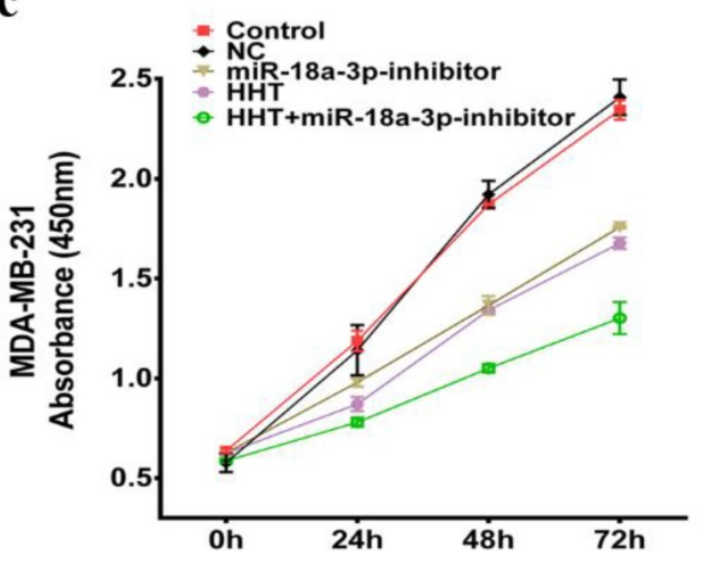

b

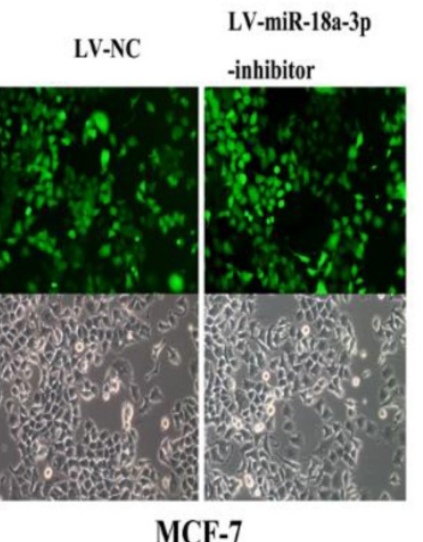

d
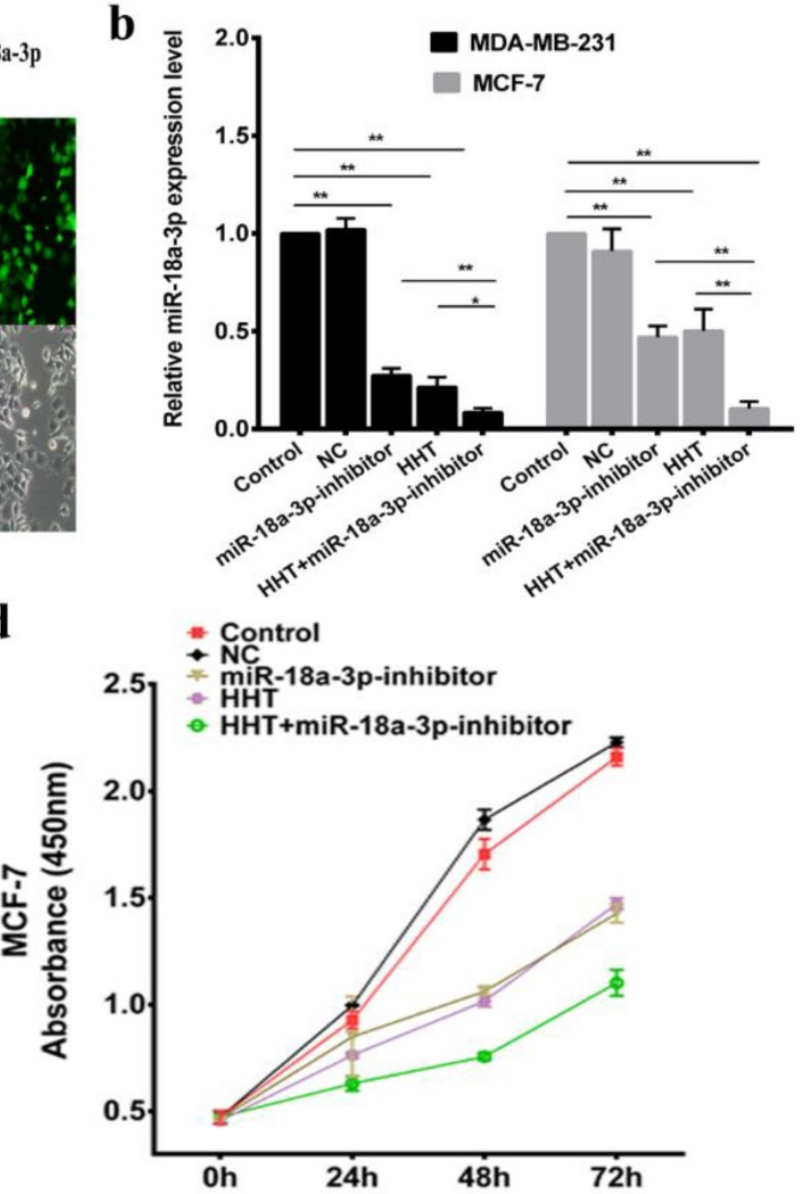

e
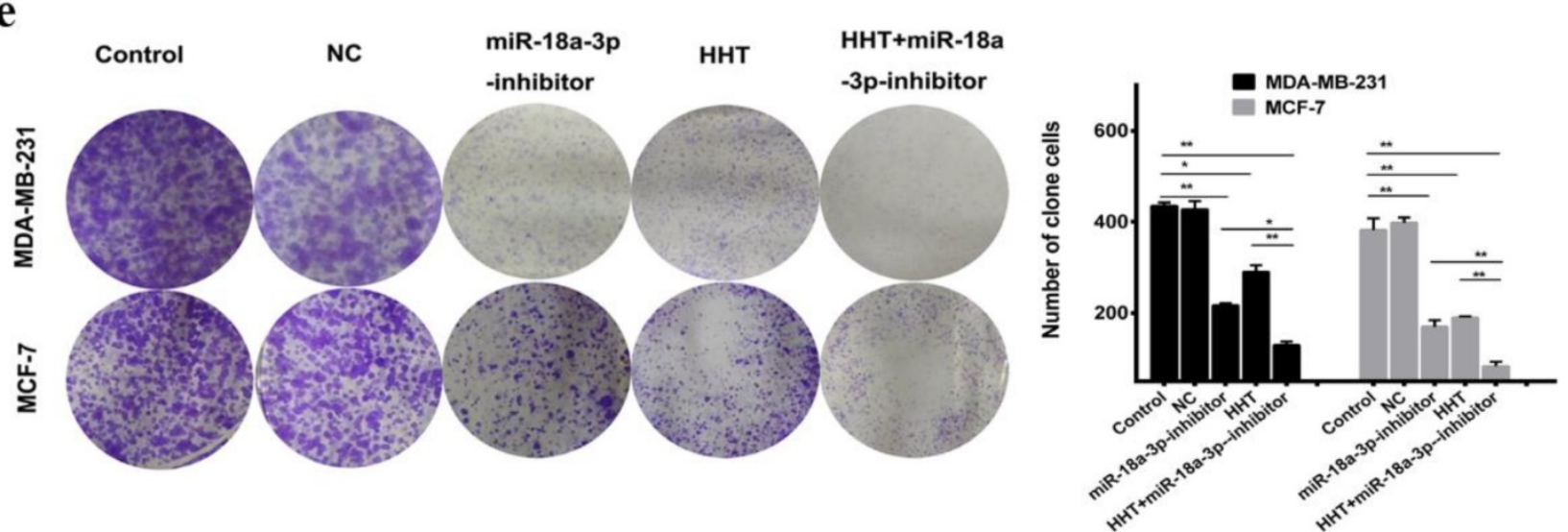

Figure 5. Downregulating miR-18a-3p increased HHT-induced inhibition of cell growth. a. Observed green fluorescence under the fluorescence microscope and verified the infection efficiency of lentivirus in both breast cancer cells. b. Validated the expression of miR-18a-3p when infected with LV- miR-18a-3p or LV-NC by qRT-PCR.The cell proliferative ability was detected by MTT (c and d) and clone formation assays (e). All data were analysed using unpaired t-tests and are shown as the means \pm SD. * $<<0.05$, $* * p<0.01$. 
were increased in the tissues treated by HHT. Meanwhile, Bcl-2 and Ki-67 expression were decreased in the group. Western blotting being used to further illucide the influence of HHT on the AKT-mTOR signaling pathway in xenografts. The results showed p-mTOR (ser-2481) and p-AKT (ser-473) were all decreased when treated with HHT, which were consistent with those of the cell treatment group.

\section{Discussion}

Breast cancer was one of the most common malignancy among women all over the world [1]. Despite significant advances therapeutic have been made, the occurrence of metastasis still leads to poor prognosis and low survival for breast cancer. Therefore, to find new medicine for breast cancer therapy has been gradually put on the agenda. Using natural compounds as anti-cancer medicines was always been applied in different cancer. Homoharringtonine (HHT), an alkaloid which extracted from cephalotaxus, have been used for hematological malignancies treatment many years.

a
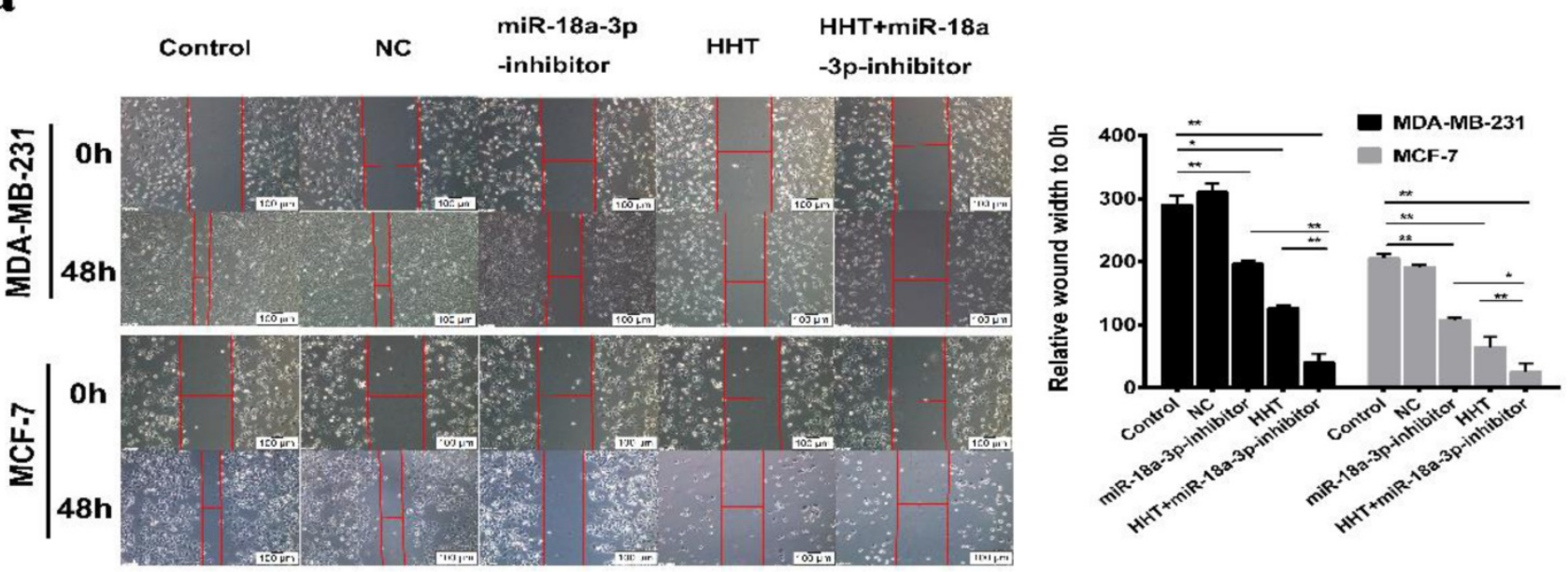

b
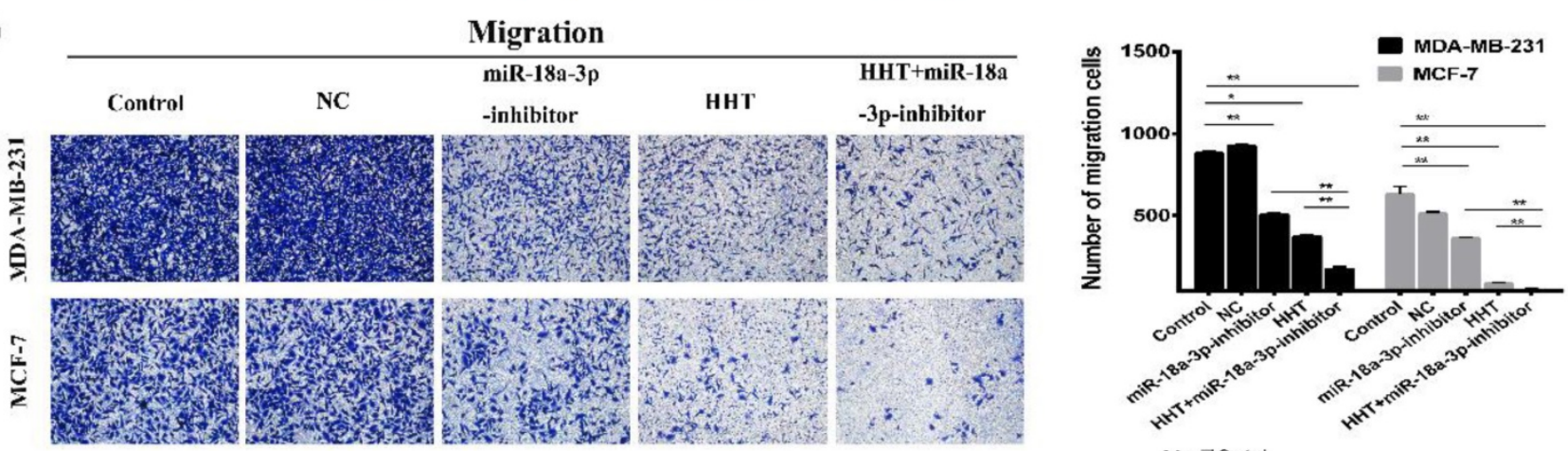

$\mathbf{C}$

MDA-MB-231

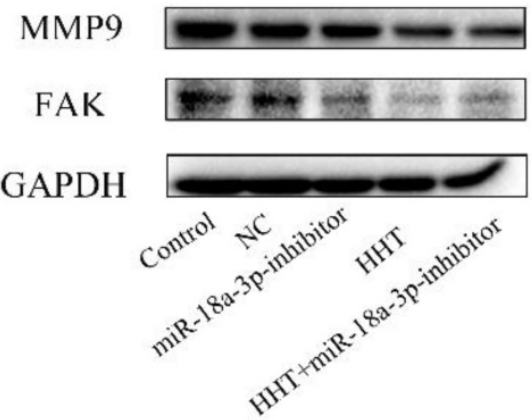

MCF-7

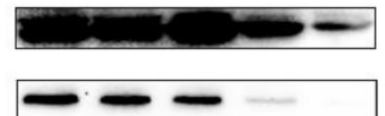

$67 \mathrm{KD}$

$110 \mathrm{KD}$
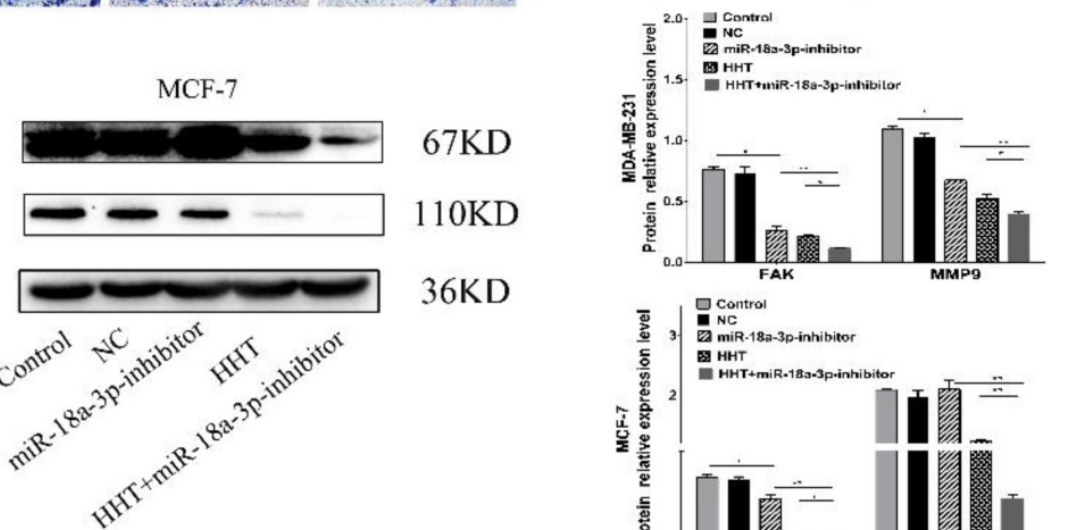

$36 \mathrm{KD}$

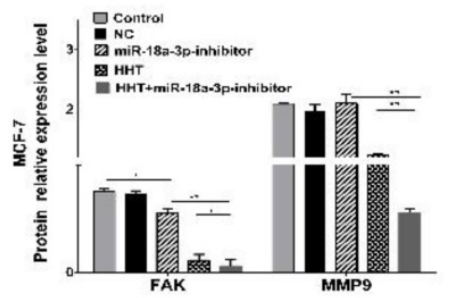

Figure 6. Downregulating miR-18a-3p increased HHT-induced inhibition of cell migration. a. Migration ability were detected using scratch wound healing assay $($ scale bar $=100 \mu \mathrm{m})$. b. Cell metastatic was assessed using transwell assay. c. The expression of MMP9 and FAKwere analysed by Western blot. GAPDH was used as the control. $* \mathrm{P}<0.05, * * \mathrm{P}<0.01$. 
a
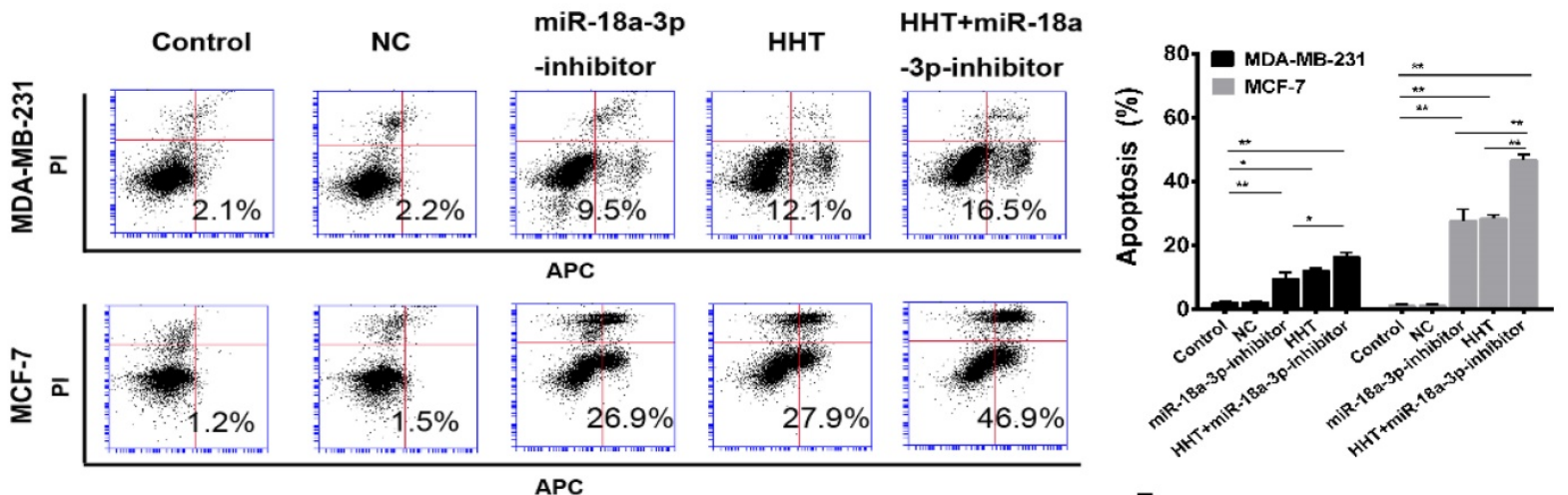

b

MDA-MB-231
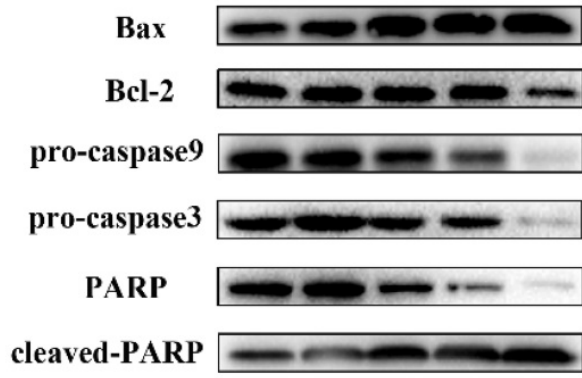

GAPDH

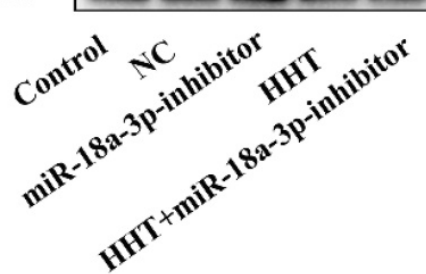

MCF-7

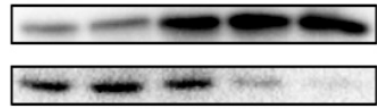

20KD

28KD

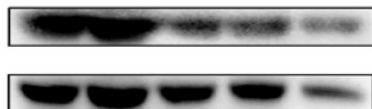

50KD

$35 \mathrm{KD}$

89KD

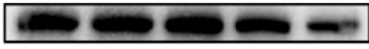

89KD

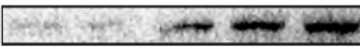

$36 \mathrm{KD}$

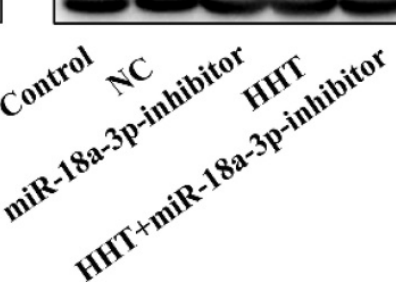

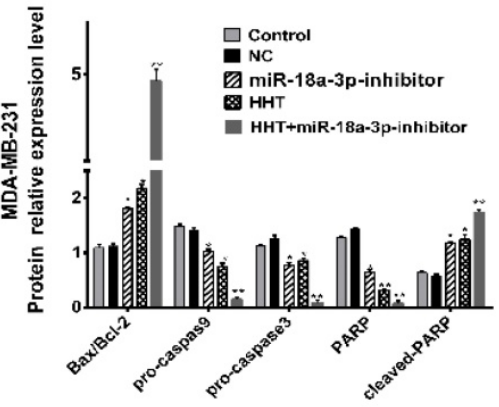

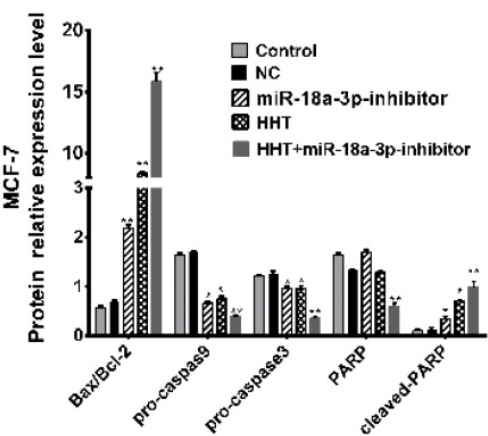

Figure 7. Downregulating miR-18a-3p increased HHT-induced cell apoptosis. a. Flow cytometry was used to detect the effect of miR-18a-3p-inhibitor on the apoptosis rate of two breast cancer cells. b. Western blot analysis of the effect of miR-18a-3p-inhibitor on the expression of apoptosis-regulated proteins in breast cancer cells.GAPDH was used as the control. All data were analysed using unpaired t-tests and are shown as the means \pm SD. ${ }^{*} \mathrm{p}<0.05$, ${ }^{* *} \mathrm{p}<0.01$.

Recent reports have shown that HHT can be used for solid tumor treatment, especially in breast cancer. Mohamad Yakhni et al. reported that HHT can inhibit tumor growth of four different types of breast cancer cells in vitro [15]. Isoharringtonine (IHT), a natural analogue of HHT, was found to have functional of decreasing the proliferation, migration, and breast cancer stem cell proportion via inhibition of the STAT3/Nanog pathway [16]. It was also reported that HHT was sensitive to the triple-negative breast cancer (TNBC) cell lines, indicating that HHT was an effective medicine for triple-negative breast cancer [17]. However, the underlying regulation mechanisms of how HHT treated breast cancer are not being fully explained. In this study, we demonstrated that HHT inhibited human breast cancer cell growth and proliferation in vivo and in vitro. Besides, HHT can also induce breast cancer cell apoptosis by regulated the expression of apoptosis-related protein like Bax/Bcl-2, Caspase3/Caspase9 and PARP.
microRNA as one a noncoding RNA, had involved in different biological processes, including cell growth, proliferation, differentiation, metastasis and drug-resistance $[18,19]$.

An increasing number of evidences indicated that miRNAs play a crucial role in cancer proliferation and metastasis [20, 21]. To further illustrate the molecular mechanism of how HHT inhibited BC proliferation, miRNA sequencing being used to analysis the PCR, 379 differentially expressed candidate miRNAs being identified. Among them, miR-18a-3p was significantly down-regulated in breast cancer cells after HHT treatment. Previous research has showed that miR-18a-3p has suppressed Dicer expression and increased Paclitaxel (PTX) resistance in TNBC cells [22]. Our research suggested that HHT could inhibit the expression of miR-18a-3p. When treated the BC cell combined with HHT and miR-18a-3p inhibitor, the cell proliferation, differentiation were all being suppressed, while 
apoptosis of the cells being promoted. The results suggested that the functions of HHT on BC cells can be enhanced by down-regulation of miR-18a-3p.

When analysis the signaling pathway related to the miR-18a-3p, we found that AKT/mTOR signaling pathway were significantly associated with miR-18a-3p. As we all know, mTOR is a key kinase which was activated by phosphoralytion of AKT. The AKT-mTOR signaling pathway could regulate cancer cell proliferation, growth, survival and angiogenesis $[23,24]$. It was reported that AKT-mTOR signaling pathway was involved in the process of HHT therapy for hematological malignancy [25, 26]. Our previous research has confimed that HHT inhibited human colorectal cancer cell proliferation and induced the cell apoptosis by blockage of mTOR signaling pathway [27]. In this study, we detected the proteins expression of mTOR signaling pathway after treated with miR-18a-3p inhibitor and HHT or both in vitro and in vivo. Bioinformatics analysis showed that there is a strong correlation between miR-18a-3p and mTOR signaling pathway. miR-18a-3p exercised its regulatory role through the AKT-mTOR signaling pathway. When treated the cell with miR-18a-3p inhibitor and HHT, the mTOR singnaling pathway expression being suppressed. The mouse xenografts experiment consisitant with the results. All the experiments proved that HHT could induce BC cell a

PDGF signaling pathway | P00047

PI3 kinase pathway | P00048

IGF pathway-protein kinase B signaling cascade | P00033

Toll receptor signaling pathway | P00054

Interferon-gamma signaling pathway | P00035

EGF receptor signaling pathway | P00018

Wnt signaling pathway | P00057

p53 pathway feedback loops 2 | P04398

Cadherin signaling pathway | P00012

S-adenosylmethionine biosynthesis | P02773

\section{Significant Enriched PANTHER.pathway Terms (Top 10)}

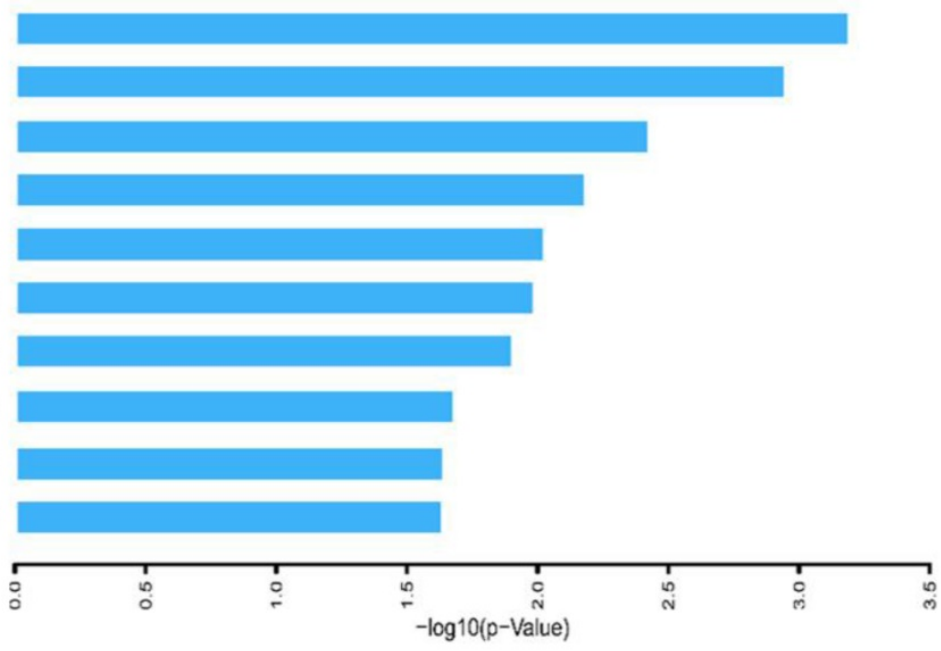

MDA-MB-231
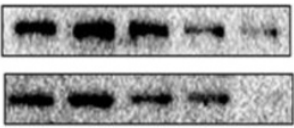

PDK1

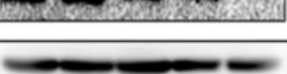

AKT

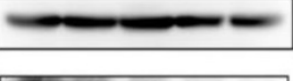

p-AKT $T^{\text {Ser-473 }}$
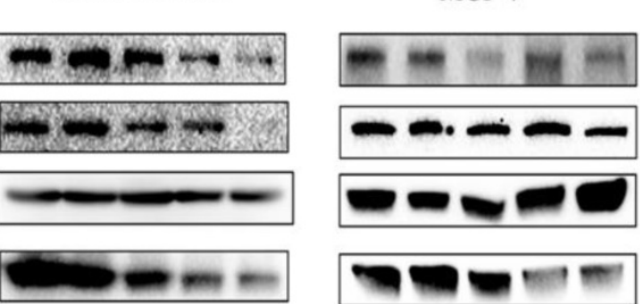

$200 \mathrm{KD}$
$68 \mathrm{KD}$
$60 \mathrm{KD}$
$60 \mathrm{KD}$

mTOR

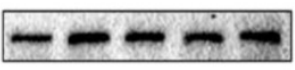

p-mTOR ${ }^{\mathrm{s} 2481}$
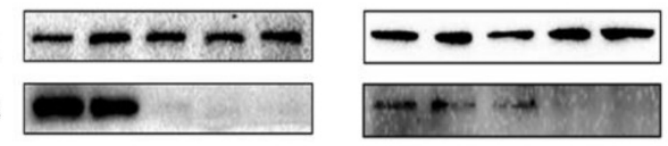

289KD

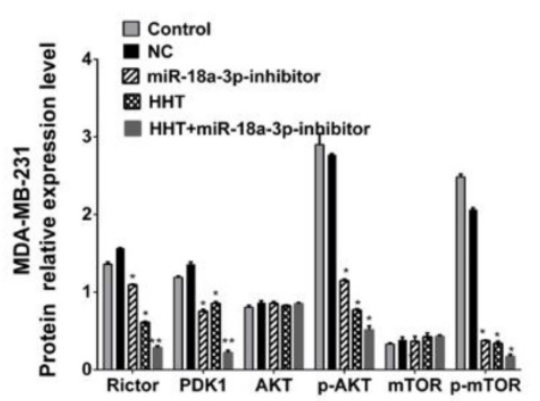

GAPDH
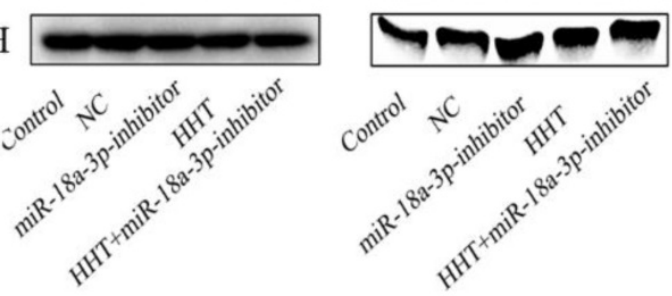

289KD

$36 \mathrm{KD}$

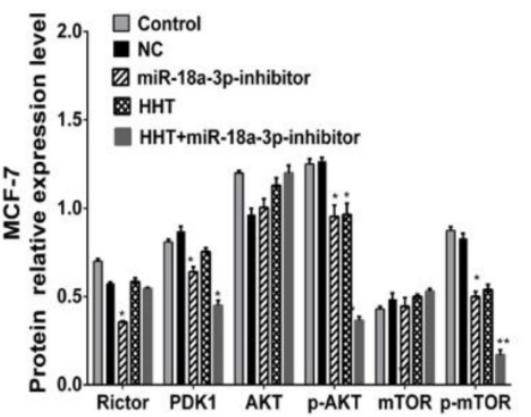

Figure 8. HHT inhibits the expression of genes related to AKT/mTOR signaling pathway. a. KEGG pathway enrichment analysis of genes downregulated (determined via whole transcriptome analyses (RNA-seq)) in both cells after treament with HHT. The top 10 signaling pathway based on fold enrichment are shown. b. Western blot analyses of Pathway related protein, including AKT, p-AKT, PDK1, mTOR and p-mTOR. GAPDH was used as the control. 
apoptosis and inhibit its proliferation by inhibition of miR-18a-3p and mTOR signaling pathway (Fig. 10).

In conclusion, our study proved that HHT suppressed breast cancer cells proliferation, migration and induced apoptosis through miR-18a-3p/AKT/ mTOR signaling pathway. We therefore confirmed that HHT exhibits a strong anti-cancer activity in breast cancer cells. HHT may have the potential to be a promising medicine for the treatment of breast cancer.

\section{Acknowledgements}

This study was funded by The Science and

a

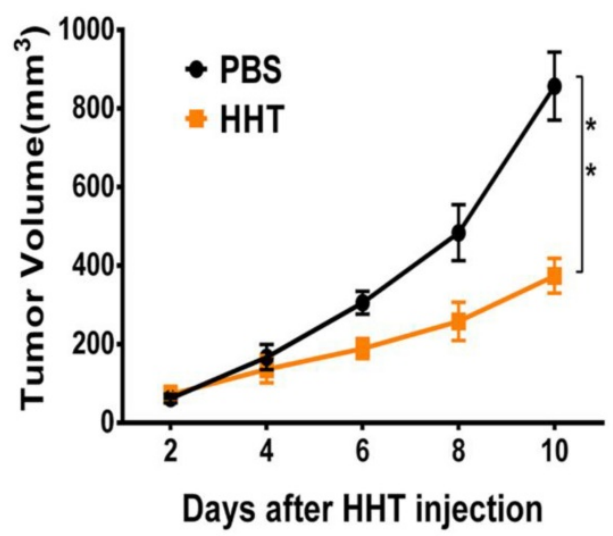

b
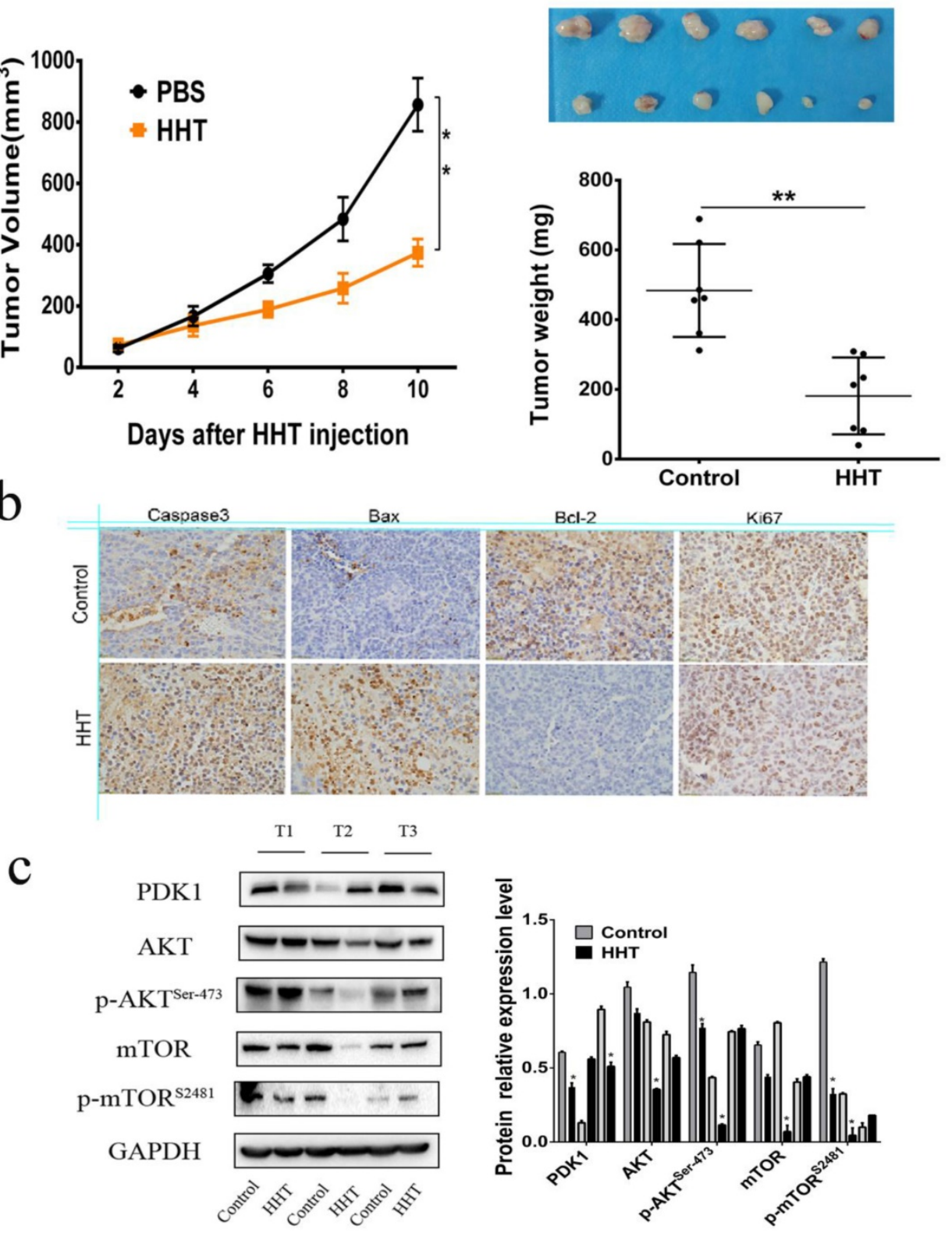

Technology Cooperation Projects of Ningxia Autonomous Region Key R\&D Programs (No. 2019BFH02012). The National Natural Science Foundation of China (No. 81860470). Ningxia High Level Science and Technology Innovation Leading Talent Project (No. KJT2019003). Ningxia Biochip Technology Research and Development Innovation Team (No. 2019-18). The Scientific Research Platform Open Project of the General Hospital of Ningxia Medical University (No. 2020-146). The Science Research Project of the General Hospital of Ningxia Medical University (No. XM2020159).

Figure 9. HHT treatment led to tumor growth slowed in vivo. a,c. Tumor diameters were measured after therapy 3 times every week, and tumor volumes were calculated. Values are mean \pm SD. *p $<0.05$. b. MCF-7 cells were injected subcutaneously into BALB/c male nude mice. After cycle of therapy, animals were sacrificed and tumors were excised. 


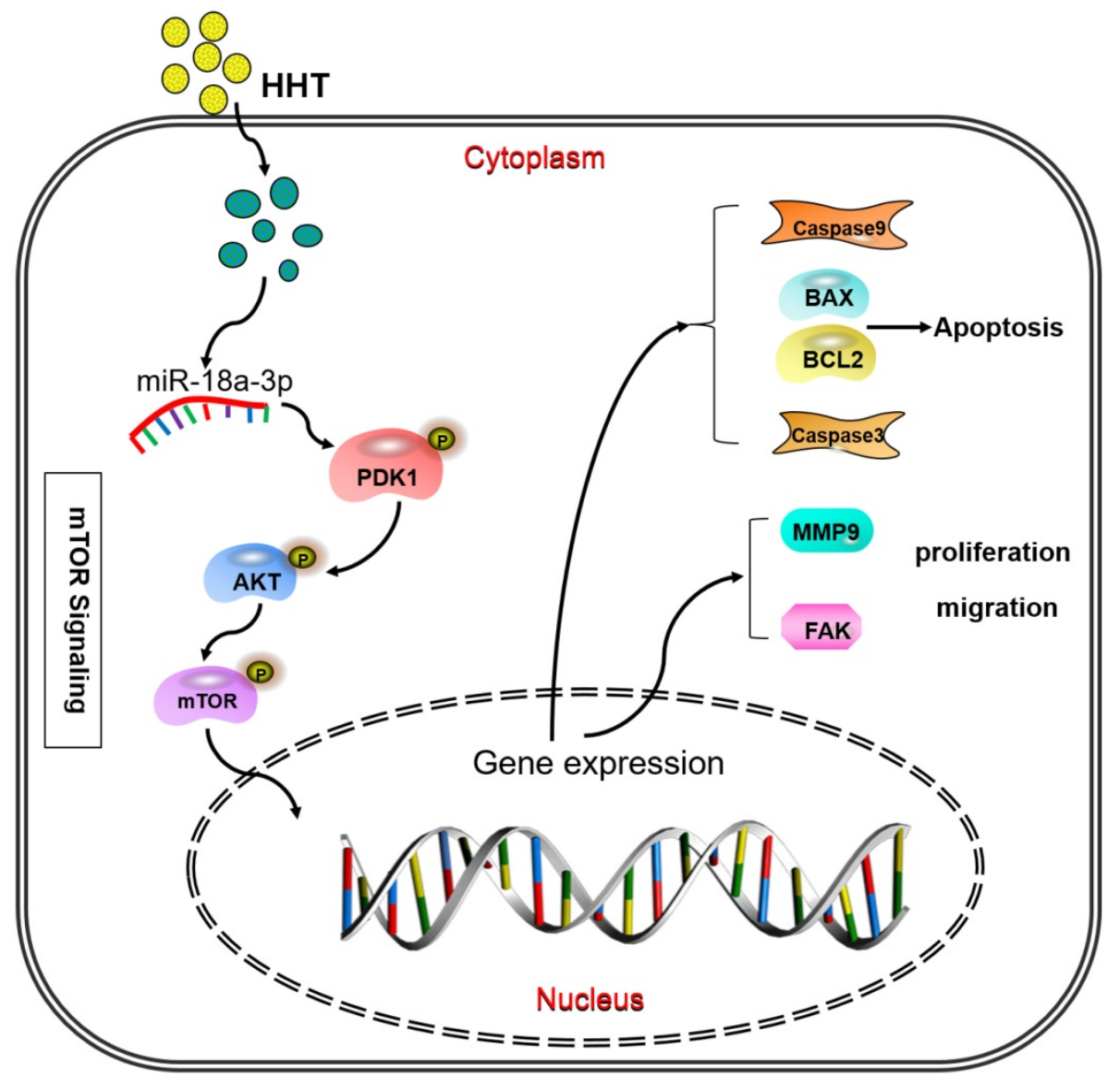

Figure 10. Pattern diagram HHT influence miR-18a-3p and AKT/mTOR signaling pathway. The pattern diagram demonstrated how HHT influence miR-18a-3p and AKT/mTOR signaling pathway expression and exhibited its function.

We thank Dr. Li-bin Wang for contributed to the conception and design of this study. The acquisition of data was carried out by Dan-ni Wang, Jia Cao, Jin-hai Tian, Rong Liu, Rong Ma, Jing-jing Yu, Jia Wang and Qi Huang. The analysis of data was carried by Dr. Wen-yong Xiong and Dr. Xu Zhang. All authors contributed to the interpretation of the results and manuscript revising and discussion critically for important intellectual content.

\section{Competing Interests}

The authors have declared that no competing interest exists.

\section{References}

1. J, Ferlay, Soerjomataram I, Dikshit R, et al. Cancer incidence and mortality worldwide: sources, methods and major patterns in GLOBOCAN 2012. International Journal of Cancer. 2015; 136(5): 359-386.

2. B, Brinkhaus, Kirschbaum B, Stöckigt B, et al. Prophylactic acupuncture treatment during chemotherapy with breast cancer: a randomized pragmatic trial with a retrospective nested qualitative study. Breast cancer Research and Treatment. 2019; 178(3): 617-628.

3. M, Nikolaou, Pavlopoulou A, Georgakilas AG, et al.The challenge of drug resistance in cancer treatment: a current overview. Clinical \& Experimental Metastasis. 2018; 35(4): 309-318.

4. X, Ma, Liang AL, and Liu YJ. Research progress on the relationship between lung cancer drug-resistance and microRNAs. Journal of Cancer. 2019; 10(27): 6865-6875.
5. M, Ito, Horimoto $Y$, Tokuda E, et al. Impact of circulating tumour cells on survival of eribulin-treated patients with metastatic breast cancer. Medical Oncology. 2019; 36(10): 89-102.

6. I, Gomes, Aguiar P, Miranda A, et al.Overall Survival of Patients With Locoregional and Metastatic Breast Cancer: Is the Influence of Baseline Characteristics the Same?. Anticancer research. 2019; 39(9): 5135-5142.

7. RM, Tujebajeva, Graifer DM, Karpova GG, et al. Alkaloid homoharringtonine inhibits polypeptide chain elongation on human ribosomes on the step of peptide bond formation. FEBS Letters. 1989; 257(2): 254-256.

8. $\mathrm{Z}, \mathrm{Wu}$, Zhuang $\mathrm{H}, \mathrm{Yu} \mathrm{O}$, et al. Homoharringtonine Combined with the Heat Shock Protein 90 Inhibitor IPI504 in the Treatment of FLT3-ITD Acute Myeloid Leukemia. Translational Oncology. 2019; 12(6): 801-809.

9. J, Kuroda, Kamitsuji Y, Kimura S, et al. Anti-myeloma effect of homoharringtonine with concomitant targeting of the myeloma-promoting molecules, Mcl-1, XIAP, and beta-catenin. International Journal of Hematology. 2008; 87(5): 507-515.

10. W, Cao, Liu Y, Zhang R, et al. Homoharringtonine induces apoptosis and inhibits STAT3 via IL-6/JAK1/STAT3 signal pathway in Gefitinib-resistant lung cancer cells. Scientific Reports. 2015; 5: 8477-8494.

11. TY, Weng, Wu HF, Li CY, et al. Homoharringtonine induced immune alteration for an Efficient Anti-tumor Response in Mouse Models of Non-small Cell Lung Adenocarcinoma Expressing Kras Mutation. Scientific Reports. 2018; 8(1): 1-11.

12. $\mathrm{Q}, \mathrm{Sun}, \mathrm{Li} \mathrm{S}, \mathrm{Li}$ J, et al. Homoharringtonine regulates the alternative splicing of Bcl-x and caspase 9 through a protein phosphatase 1-dependent mechanism. BMC Complementary and Altern Med. 2018; 18(1): 1-11.

13. C, Damaskos, Garmpi A, Nikolettos K, et al.Triple-Negative Breast Cancer: The Progress of Targeted Therapies and Future Tendencies. Anticancer Research. 2019; 39(10): 5285-5296.

14. V, Sharma, Sharma AK, Punj V, et al. Recent nanotechnological interventions targeting PI3K/Akt/mTOR pathway: A focus on breast cancer. Seminars in Cancer Biology, 2019. 59: 133-146.

15. M, Yakhni, Briat A, El Guerrab A, et al. Homoharringtonine, an approved anti-leukemia drug, suppresses triple negative breast cancer growth through a rapid reduction of anti-apoptotic protein abundance. American Journal of Cancer Research. 2019; 9(5): 1043-1060. 
16. W, Chen, Wang $\mathrm{H}$, Cheng $\mathrm{M}$, et al. Isoharringtonine inhibits breast cancer stem-like properties and STAT3 signaling. Biomed Pharmacother. 2018;103:435-442.

17. R, Tang, Faussat AM, Majdak P, et al. Semisynthetic homoharringtonine induces apoptosis via inhibition of protein synthesis and triggers rapid myeloid cell leukemia-1 down-regulation in myeloid leukemia cells. Molecular Cancer therapeutics. 2006; 5(3): 723-731.

18. WM, Sun, Tao W, Li JC, et al. MicroRNA-296 functions as a tumor suppressor in breast cancer by targeting FGFR1 and regulating the Wnt/ $\beta$-catenin signaling pathway. European Review for Medical and Pharmacological Sciences. 2019; 23(23): 10422-10432.

19. C, Noronha Fernandes-Brum, Marinho Rezende P, Cherubino Ribeiro TH, et al. A genome-wide analysis of the RNA-guided silencing pathway in coffee reveals insights into its regulatory mechanisms. PloS One. 2017;12(4): 1-29.

20. M Shen, Dong C, Ruan X, et al. Chemotherapy-Induced Extracellular Vesicle miRNAs Promote Breast Cancer Stemness by Targeting ONECUT2. Cancer Res. 2019; 79(14):3608-3621.

21. R, Gernapudi, Yao Y, Zhang $Y$, et al. Targeting exosomes from preadipocytes inhibits preadipocyte to cancer stem cell signaling in early-stage breast cancer. Breast Cancer Research and Treatment. 2015;150(3): 685-695.

22. LY, Sha, Zhang Y, Wang W, et al. MiR-18a upregulation decreases Dicer expression and confers paclitaxel resistance in triple negative breast cancer. European Review for Medical and Pharmacological Sciences, 2016. 20(11): 2201-2208.

23. H, Kooshapur, Choudhury NR, Simon B, et al. Structural basis for terminal loop recognition and stimulation of pri-miRNA-18a processing by hnRNP A1. Nature Communications. 2018; 9(1): 1-17.

24. H, Zhou, $\mathrm{Xu}$ RZ, Gu Y, et al. Targeting of phospho-eIF4E by homoharringtonine eradicates a distinct subset of human acute myeloid leukemia. Leukemia \& Lymphoma.2020; 61(5):1084-1096.

25. P, Chen, Wen X, Wang B, et al. PI3K/Akt inhibitor LY294002 potentiates homoharringtonine antimyeloma activity in myeloma cells adhered to stromal cells and in SCID mouse xenograft. Annals of Hematology. 2018; 97(5): 865-875.

26. H, Meng, Yang C, Jin J, et al. Homoharringtonine inhibits the AKT pathway and induces in vitro and in vivo cytotoxicity in human multiple myeloma cells. Leukemia \& Lymphoma. 2008; 49(10): 1954-1962.

27. D, Wang, Tao $W$, Zhang $X$, et al. Blockage of mTOR signaling pathway by homoharringtonine inhibits proliferation and induces apoptosis of HT29 human colorectal tumor cells. Chinese Journal of Cellular and Molecular Immunology. 2018;34(4): 346-353. 